\title{
Monopolar and bipolar auroral electric fields and their effects
}

\author{
J. De Keyser, R. Maggiolo, and M. Echim \\ Belgian Institute for Space Aeronomy (BIRA-IASB), Ringlaan 3, 1180 Brussels, Belgium
}

Received: 30 June 2010 - Revised: 13 September 2010 - Accepted: 27 October 2010 - Published: 9 November 2010

\begin{abstract}
Most of the high-altitude auroral electric fields observed by CLUSTER can be classified into monopolar and bipolar structures. The observations associate monopolar electric fields with polar cap boundary arcs, while bipolar fields tend to be linked to discrete arcs within the auroral oval and to polar cap arcs. The present paper proposes an explanation for this association based on a simple model of the magnetotail configuration and kinetic model computations. The paper introduces a quasi-electrostatic model to describe the auroral current system associated with monopolar and bipolar high-altitude fields. Analytic solutions are presented. The model gives indications about the location of the up- and downward field-aligned current regions, the ionospheric and magnetospheric convection along the arc, the acceleration or deceleration of precipitating particles, and the behaviour of escaping ionospheric ions.
\end{abstract}

Keywords. Magnetospheric physics (Auroral phenomena; Current systems; Magnetosphere-ionosphere interactions)

\section{Introduction}

Johansson et al. $(2005,2006)$ have conducted an in-depth survey of the high-altitude electric fields that have been observed by CLUSTER above the auroral regions. Flying at an altitude of 4-6 $R_{\mathrm{E}}$, CLUSTER indicates that strong auroral electric fields are always found at plasma boundaries in the magnetosphere. The observed electric field profiles can have various forms. One way of classifying these structures is to distinguish "convergent" and "divergent" electric fields, which correspond to V-shaped or nested V-shaped magnetospheric potential profiles (e.g., Lyons, 1980, 1981; De Keyser and Echim, 2010). Another classification considers three

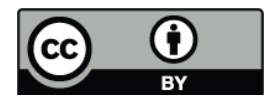

Correspondence to: J. De Keyser (johan.dekeyser@aeronomie.be) groups: electric field profiles that are monopolar, bipolar, or of a more complicated nature. Johansson et al. (2006) have related monopolar fields to the lobe-plasma sheet interface, and thus to polar cap boundary arcs, while bipolar electric fields tend to be associated with interfaces within the plasma sheet. Bipolar electric fields are also found in polar cap arcs (Maggiolo et al., 2006).

The present paper examines auroral monopolar and bipolar electric fields in the context of kinetic descriptions of magnetospheric boundary layers that might be the generators of discrete arcs or other auroral phenomena (Roth et al., 1996; De Keyser, 1999; Echim et al., 2008). The paper also addresses the general properties of the auroral current circuits associated with such monopolar and bipolar electric fields. These can be understood in terms of the structures identified for convergent/divergent electric fields (De Keyser and Echim, 2010), since monopolar and bipolar electric field profiles can be regarded as a succession of convergent and/or divergent electric field configurations. Because of their frequent occurrence in the auroral regions, however, monopolar and bipolar fields merit a more detailed study.

Special attention is given to the high-altitude signatures of the auroral system that are observable by spacecraft such as CLUSTER: The high-altitude perpendicular electric field, the field-aligned currents, the upgoing electrons, and the escaping ionospheric ions. The latter, in particular, turn out to be sensitive indicators of auroral current system structure. They are also important in their own right, as auroral ion escape is a major source of heavy ions in the terrestrial magnetosphere.

This paper is organized as follows. Section 2 describes the general configuration and proposes a qualitative explanation as to why polar cap boundary arcs are associated with highaltitude monopolar fields while auroral oval arcs and polar cap arcs tend to correspond to high-altitude bipolar electric fields. Section 3 focuses on the auroral current system of monopolar electric fields. Section 4 presents a similar analysis for the case of bipolar fields. The paper concludes with

Published by Copernicus Publications on behalf of the European Geosciences Union. 
Table 1. Notation.

\begin{tabular}{|c|c|}
\hline Symbol & Meaning \\
\hline$\rho_{s}$ & gyroradius of (thermal) particles of species $s$ \\
\hline$L_{S}, \ell_{S}$ & absolute and dimensionless transition length of species $s$ in a TD interface \\
\hline$W_{\perp s}$ & perpendicular energy of (thermal) particles of species $s$ \\
\hline$h$ & altitude of the topside ionosphere \\
\hline$x$ & ionospheric coordinate across a 1-D structure, measured at altitude $h$ \\
\hline$\hat{x}$ & magnetospheric coordinate across a 1-D structure, measured at high altitude \\
\hline$b$ & scaling factor relating distances at magnetospheric and ionospheric altitude \\
\hline$\Delta_{\perp} \phi_{\text {tail }}$ & electric potential difference across the magnetotail \\
\hline$\Delta_{\perp} \phi_{\text {ext }}$ & electric potential difference imposed across a TD interface \\
\hline$\Delta_{\perp} \phi_{\mathrm{TD}}$ & maximum electric potential difference supported by a TD interface \\
\hline$\phi$ & ionospheric electric potential at altitude $h$ \\
\hline$\hat{\phi}$ & magnetospheric electric potential at high altitude \\
\hline$\Delta_{\perp} \hat{\phi}, \Delta_{\perp} \hat{\phi}_{\mathrm{L}}, \Delta_{\perp} \hat{\phi}_{\mathrm{R}}$ & electric potential differences across magnetospheric interfaces \\
\hline$\epsilon_{\mathrm{L}}, \epsilon_{\mathrm{R}}, \epsilon_{\mathrm{C}}$ & magnetospheric convection electric fields mapped to altitude $h$ \\
\hline$\Delta_{\|} \phi$ & field-aligned potential difference between ionosphere and magnetosphere \\
\hline$j_{\|}$ & field-aligned current (FAC) density at altitude $h$ \\
\hline$\hat{j}_{\|}$ & field-aligned current density at high altitude \\
\hline$J_{\downarrow}, J_{\uparrow}$ & total downward and upward currents \\
\hline$\Sigma_{\mathrm{P}}, I_{\mathrm{P}}$ & height-integrated Pedersen conductivity and Pedersen current in the ionosphere \\
\hline$P, P_{\mathrm{L}}, P_{\mathrm{R}}$ & half-thickness of magnetospheric interfaces, measured at altitude $h$ \\
\hline$S$ & half-thickness of embedded plasma region, measured at altitude $h$ \\
\hline$\xi, \eta$ & positions where $\Delta_{\|} \phi$ changes sign, measured at altitude $h$ \\
\hline$K_{+}$ & Knight constant for the upward current in the basic FAC model \\
\hline$K_{-}$ & Knight constant for the return current above the polar cap \\
\hline$K_{*}$ & Knight constant for the return current above the oval in the basic FAC model \\
\hline$\lambda_{+}, \lambda_{-}, \lambda_{*}$ & length scales associated with $K_{+}, K_{-}, K_{*}$, measured at altitude $h$ \\
\hline$j_{\mathrm{PO}}$ & precipitation/outflow offset current in the advanced FAC model \\
\hline$j_{0}$ & field-aligned current for $\Delta_{\|} \phi=0$ in the advanced FAC model \\
\hline$K_{0}$ & Knight constant for the precipitation/outflow offset current \\
\hline$\tilde{\lambda}_{+}, \tilde{\lambda}_{*}$ & length scales associated with $K_{+}+K_{0}$ resp. $K_{*}+K_{0}$, measured at altitude $h$ \\
\hline$\Delta \phi_{+}, \Delta \phi_{*}$ & equivalent electric potentials associated with $j_{0}$ and $K_{+}+K_{0}$ resp. $K_{*}+K_{0}$ \\
\hline
\end{tabular}

a summary of the main findings and their implications. For convenience, Table 1 gives an overview of the notation used in this paper.

\section{Global configuration}

At the outset, it is assumed that the magnetosphere harbours sufficiently long-lived electric potential structures that drive auroral current systems, i.e., the quasi-electrostatic picture is adopted here (as done by Lyons, 1980, 1981; Roth et al., 1996; De Keyser, 1999; Echim et al., 2008; De Keyser and Echim, 2010). Such magnetospheric potential structures can act as generators and are connected via field-aligned currents to the ionosphere, which behaves as a load. It is, however, also possible that the ionosphere at times acts as a driver of the electric circuit. The model described here applies to both cases. The central assumption is that the feedback effects of the currents on the ionosphere and on the magnetospheric potential structure are not too strong so that the system is in a quasi-static state. This assumption is believed to be realistic for long-lived larger-scale auroral structures, although it cannot be true in general because of the dynamic auroral environment. The analysis presented here therefore refers to an idealization, which nevertheless bears out a number of features observed in aurora (Vaivads et al., 2003; Echim et al., 2009).

\subsection{Electric potential differences in the tail}

Models of the solar wind-magnetosphere interaction imply the existence of a dawn-dusk electric field across the magnetotail, corresponding to a cross-tail potential difference $\Delta_{\perp} \phi_{\text {tail }}$. The cold plasma in the lobe convects along equipotential lines as its motion is dictated by the $\boldsymbol{E} \times \boldsymbol{B}$ drift. The convection patterns for different IMF conditions have been reconstructed from CLUSTER measurements (Haaland et al., 2008). They are reflected by the corresponding cross-polar cap potential and the convection cell pattern in the ionosphere. The cross-tail potential is typically on the order of 
$40 \mathrm{kV}$. It is therefore not surprising that electric potential differences of a fraction of $\Delta_{\perp} \phi_{\text {tail }}$ would exist across the plasma sheet boundary, as this is the boundary between open field lines connecting through the lobe to the solar wind, and closed field lines that thread the plasma sheet, especially during substorms when the configuration is dynamically changing. There is, indeed, no a priori reason why the electric potential on either side of that boundary would be identical.

\subsection{Properties of discontinuities in the tail}

In a simplified picture, there exist two kinds of plasmas in the tail: cold and hot plasma. Cold plasma fills the lobes. While originally thought to be characterized by particle densities of $0.01 \mathrm{~cm}^{-3}$ (e.g. Gosling et al., 1985), densities are now believed to be $0.1-0.5 \mathrm{~cm}^{-3}$ (Engwall et al., 2006). Detection of the lobe plasma is difficult because of its low energy (a few $\mathrm{eV})$. Hot plasma fills the central plasma sheet and the plasma sheet boundary, with densities up to a few particles per $\mathrm{cm}^{3}$ and ion energies on the order of $5-10 \mathrm{keV}$. These distinct plasmas are separated by field-aligned interfaces; the magnetic field lines in the lobes are essentially parallel tailward from about $10 R_{\mathrm{E}}$. One can therefore think about plasma sheet structure in the tail as being of the tangential discontinuity (TD) type in a rough first approximation. The kinetic structure of such discontinuities has been modeled in considerable detail (see Roth et al., 1996, for a review). For the sake of simplicity of the argument, we assume here that there is no significant perpendicular plasma velocity shear across the TD.

An essential characteristic of TD layers is that there is a limit to the potential difference they can support. Indeed, consider a TD with thickness $D$ separating two plasmas with Maxwellian velocity distributions. If an external potential difference $\Delta_{\perp} \phi_{\text {ext }}$ exists across that layer, the average electric field in the layer is $E \approx-\Delta_{\perp} \phi_{\mathrm{ext}} / D$. Consider now the forces acting on a particle with charge $Z e$ and perpendicular thermal velocity $v_{\perp}$ inside the layer. The Lorentz force that lets this particle gyrate in a magnetic field with strength $B$ has magnitude $Z e B v_{\perp}$, while the external electric field inside the layer provides a force with magnitude $Z e \Delta_{\perp} \phi_{\mathrm{ext}} / D$. An equilibrium can exist only if the Lorentz force is stronger than the external electric field; otherwise the particles can no longer be tied to the field line. This condition is satisfied when

$\Delta_{\perp} \phi_{\mathrm{ext}}<v_{\perp} B D \equiv \Delta_{\perp} \phi_{\mathrm{TD}}$

The thickness of a TD in the plasma sheet must be on the order of the largest gyroradius involved, that of the hot ions, $\rho_{+}$, so that

$D=\ell \rho_{+}=\ell \frac{m_{+} v_{\perp+}}{Z e B}$ with a dimensionless thickness parameter $\ell \geq 1$, and where $m_{+}$and $v_{\perp+}$ denote ion mass and perpendicular thermal velocity. Condition (1) can then be rewritten as

$\Delta_{\perp} \phi_{\mathrm{ext}}<\Delta_{\perp} \phi_{\mathrm{TD}}=\frac{\ell}{Z e} m_{+} v_{\perp+}^{2}=\frac{2 \ell}{Z e} W_{\perp+}$,

with $W_{\perp+}=m_{+} v_{\perp+}^{2} / 2$, which tells us that the maximum electric potential difference expressed in Volt must be of the order of the energy $W_{\perp+}^{\prime}=W_{\perp+} / e$ of the hot ions expressed in electronVolt. While this simple argument does not include the polarization and shielding effects that would occur in a real TD because of the interplay between the different particle populations, it captures an essential physical ingredient that is present in fully kinetic TD models. Note that this maximum potential difference is closely related to the wellknown flow shear limit across a TD, since flow shear generates a potential difference across a finite-thickness TD layer (Sestero, 1964, 1966; Roth et al., 1996; De Keyser et al., 1997; De Keyser, 1999).

An additional feature of TDs is the structure of their internal electric field. Figure 1 presents the interface structure computed with a kinetic TD model (Roth et al., 1996). In such models, each species $s$ is characterized by a transition length scale $L_{s}=\ell_{s} \rho_{s}$, where $\rho_{s}$ is the gyroradius and $\ell_{s} \geq 1$ a dimensionless factor. The $\ell_{s}$ are free parameters in the model, but physical considerations suggest that $\rho_{+} \leq L_{+}<5 \rho_{+}$and $1<L_{+} / L_{-}<10$; in the examples below, this is achieved with $\ell_{+}=1$ and $\ell_{-}=40$. Consider an interface between a cold lobe plasma (with a density of $0.1 \mathrm{~cm}^{-3}, 1 \mathrm{eV}$ electrons and $5 \mathrm{eV}$ protons) and a hot plasma sheet plasma (with typical density $0.5 \mathrm{~cm}^{-3}, 1 \mathrm{keV}$ electrons and $5 \mathrm{keV}$ protons), with gyroradii such that $L_{\text {cold }-}<$ $L_{\text {cold }+} \ll L_{\text {hot }-}<L_{\text {hot }+ \text {. }}$ The transition of the hot particle densities across the TD takes place on the two different length scales $L_{\text {hot- }}$ and $L_{\text {hot }+ \text {, which tends to produce }}$ a charge separation effect and an attendant polarization electric field. As the length scale is rather large, this gives rise to significant potential variations. The lobe ions and electrons experience a similar charge separation effect but the resulting polarization electric field is negligible since the distance scales $L_{\text {cold }-}$ and $L_{\text {cold }+}$ are much shorter.

If there is no external potential difference $\left(\Delta_{\perp} \phi_{\text {ext }}=0\right.$, Fig. 1b) across the interface, there is a broad region with small $\left|E_{x}\right|$ (thickness scale $L_{\text {hot }+ \text { ) and a narrow region with }}$ large $\left|E_{x}\right|$ in the opposite sense (thickness on the order of $L_{\text {hot- }}$ ), so that integrating $E_{x}$ produces the net zero potential difference: This is a bipolar electric field structure. The presence of a significant electric potential difference across the layer affects the balance of positive and negative charges in the transition. For instance, for $\Delta_{\perp} \phi_{\text {ext }}<0$ (Fig. 1a), the external potential attracts hot electrons to the center of the layer and repels the hot protons, while it does the reverse for the cold plasma species. As a consequence, the electric field becomes more intense and has definitely a monopolar character. For $\Delta_{\perp} \phi_{\text {ext }}>0$ (Fig. 1c), the hot protons are 
(a) $\Delta_{\perp} \phi_{\text {ext }}=-1 \mathrm{kV}$
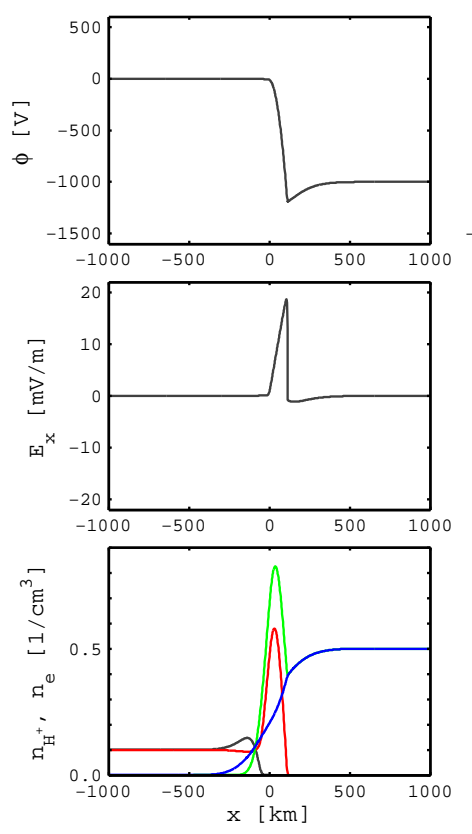

(d) $\Delta_{\perp} \phi_{\text {ext }}=-1 \mathrm{kV}$
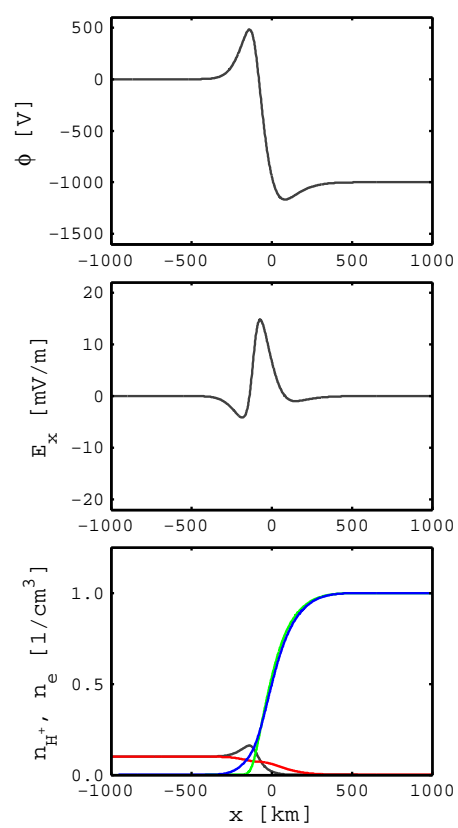

(b) $\Delta_{\perp} \phi_{\text {ext }}=0$
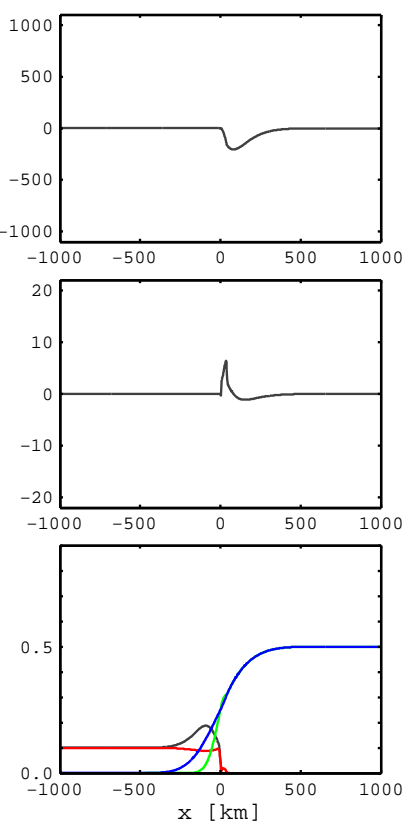

(e) $\Delta_{\perp} \phi_{\text {ext }}=0$
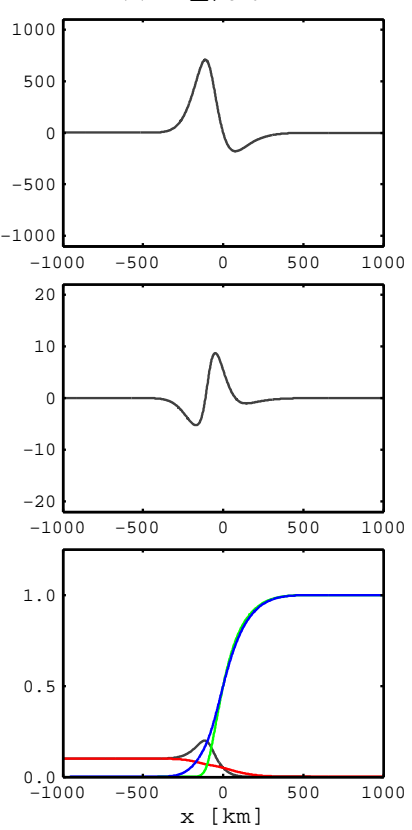

(c) $\Delta_{\perp} \phi_{\text {ext }}>+1 \mathrm{kV}$
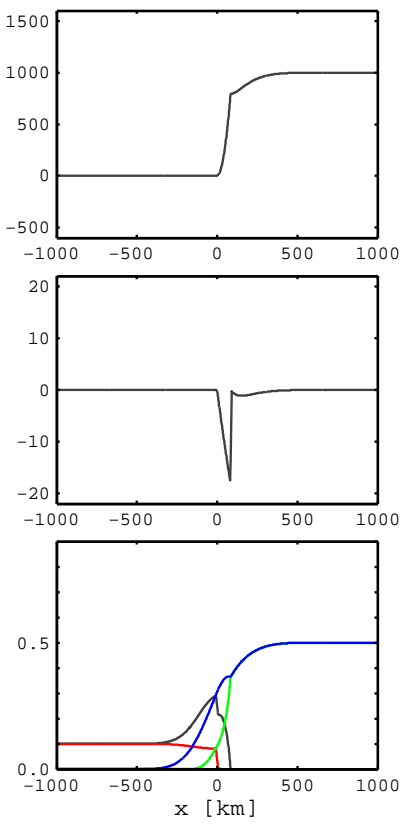

(f) $\Delta_{\perp} \phi_{\text {ext }}>+1 \mathrm{kV}$
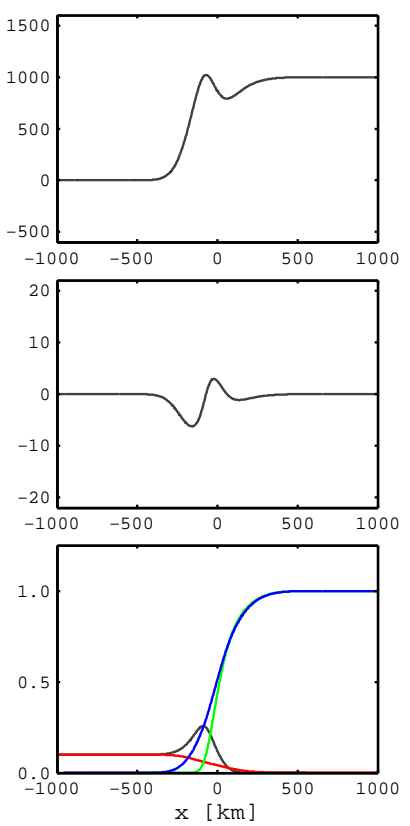

Fig. 1. Electric structure of magnetospheric tangential discontinuity interfaces for different transverse electric potential differences $\Delta_{\perp} \phi_{\text {ext }}$, as computed with a kinetic model (Roth et al., 1996). The magnetic field at the center of the interfaces was taken to be $40 \mathrm{nT}$. Top three rows (a), (b), (c): Interfaces between a cold plasma $\left(0.1 \mathrm{~cm}^{-3}, 1 \mathrm{eV}\right.$ electrons and $5 \mathrm{eV}$ protons, left of the interface) and a hot plasma $\left(0.5 \mathrm{~cm}^{-3}, 1 \mathrm{keV}\right.$ electrons and $5 \mathrm{keV}$ protons, right of the interface), typical of the lobe-plasma sheet boundary. Bottom three rows (d), (e), (f): Interfaces between two hot plasmas $\left(0.1\right.$ and $1 \mathrm{~cm}^{-3}, 1 \mathrm{keV}$ electrons and $5 \mathrm{keV}$ protons). The electric potential $\phi$, the transverse electric field $E_{x}$, and the partial densities $n_{\mathrm{e}}$ of the electrons and $n_{\mathrm{H}^{+}}$of the protons to the left (black and red) and to the right of the interface (green and blue), are given for each case.

attracted while the hot electrons are repelled; the resulting electric field structure is also monopolar, now with the opposite sense. Considering an interface within the plasma sheet, between two hot plasmas with the same temperatures ( $1 \mathrm{keV}$ electrons and $5 \mathrm{keV}$ protons) but with different densities, $0.1 \mathrm{~cm}^{-3}$ on one side and $1 \mathrm{~cm}^{-3}$ on the other side, one 

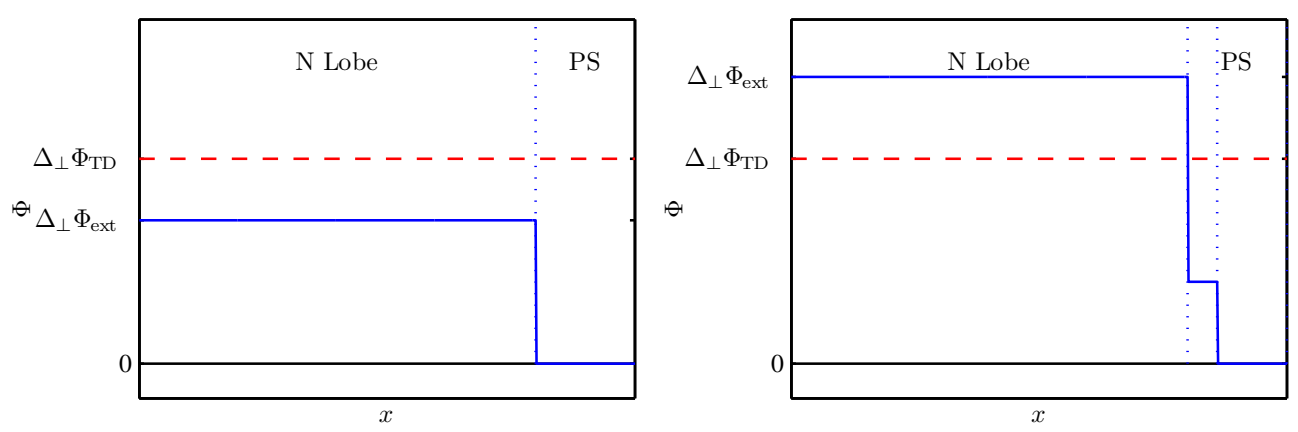

Fig. 2. Electric potential variation from the lobe to the center of the plasma sheet. Given an externally applied potential difference $\Delta_{\perp} \phi_{\text {ext }}$, and given a maximum for the potential difference $\Delta_{\perp} \phi_{\mathrm{TD}}$ across a single discontinuity, this can be accounted for by a single discontinuity when $\Delta_{\perp} \phi_{\text {ext }}<\Delta_{\perp} \phi_{\mathrm{TD}}$ (left panel) but requires more discontinuities when $\Delta_{\perp} \phi_{\text {ext }}$ is larger (right panel).

finds electric field profiles that are tripolar, but that would observationally often be considered as bipolar. Figures 1df show that the electric field exhibits structure on the two length scales $L_{\text {hot- }}$ and $L_{\text {hot+ }}$. The detailed structure depends on the degree of asymmetry between the densities on either side, and on the actual temperatures. Bipolar electric fields can of course also appear when two interfaces with monopolar electric field are situated very close together. That is the likely situation in polar cap arcs, which can often be thought of as a layer of hot plasma embedded in a colder environment (Maggiolo et al., 2006).

\subsection{Required number of interfaces}

It is clear that a transverse potential difference $\Delta_{\perp} \phi_{\text {ext }}$ between the lobe and the center of the plasma sheet may exist, depending on the global magnetospheric configuration. Since the lobe plasma is so cold, interfaces inside the lobes cannot support any significant electric potential difference. Part of the total $\Delta_{\perp} \phi_{\text {ext }}$ can be supported by lobe convection, but the remainder must appear concentrated over a number of interfaces at the lobe-plasma sheet boundary and/or inside the plasma sheet. This is illustrated in Fig. 2, where the role of lobe convection is not included for the sake of simplicity. Because of the maximum potential difference $\Delta_{\perp} \phi_{\mathrm{TD}}$ across an individual interface (Eq. 2), it follows that there must be at least

$N_{\mathrm{TD}} \geq \frac{\Delta_{\perp} \phi_{\mathrm{ext}}}{\Delta_{\perp} \phi_{\mathrm{TD}}} \approx \frac{\Delta_{\perp} \phi_{\mathrm{ext}}}{2 W_{\perp \text { hot }}^{\prime}}$

interfaces, given that usually $Z_{\text {hot }+}=1$ and $\ell_{\text {hot }+} \approx 1$. As long as the potential difference is not too large, $\Delta_{\perp} \phi_{\text {ext }}<$ $\Delta_{\perp} \phi_{\mathrm{TD}}$, there should be at least one interface $\left(N_{\mathrm{TD}} \geq 1\right)$. The left panel in Fig. 2 illustrates this case. The simplest configuration of the plasma sheet would be one in which the only hot-cold transition with a sharp density difference is located at the lobe-plasma sheet interface, thereby producing a monopolar electric field profile. Of course, the plasma sheet might have more internal structure so that there are additional interfaces inside the plasma sheet. The potential differ- ence across the plasma sheet boundary then needs not be as big (but would still produce a monopolar field) as additional smaller potential differences appear across internal plasma sheet interfaces; the latter may have monopolar or bipolar or more complicated electric fields. If the lobe-plasma sheet potential difference $\Delta_{\perp} \phi_{\text {ext }}$ is larger, additional interfaces inside the plasma sheet are necessary for an equilibrium configuration to exist. One can therefore conclude that there often must be significant potential differences across the lobeplasma sheet interfaces, leading to monopolar electric fields. At the same time, the existence of additional interfaces inside the plasma sheet should come as no surprise, and such interfaces may have monopolar, bipolar, or more complicated electric field structures, depending on the changes in temperature and density. Finally, there is also the possibility of hot plasma embedded in the lobe, giving rise to bipolar electric field structures that are pairs of monopolar fields.

\section{Monopolar electric fields}

The auroral current circuit can be studied with a onedimensional model based on current continuity. Let $x$ denote the horizontal coordinate perpendicular to the structure, measured at an altitude $h$ corresponding to the topside ionosphere, positive in the poleward direction. Height $h$ is chosen so that all horizontal currents are situated below that altitude. A high-altitude position $\hat{x}$ in the magnetosphere can be associated with each $x$ by following the magnetic field line that connects the ionosphere to the magnetosphere; the function $\hat{x}(x)$ represents this mapping. All spatial variations can then be expressed in terms of the ionospheric coordinate $x$. The high altitudes considered in this paper correspond to 5-6 $R_{\mathrm{E}}$, above the auroral acceleration region, the altitudes at which the CLUSTER spacecraft typically cross the auroral field lines. The electric potential profile in the magnetospheric generator, $\hat{\phi}(\hat{x}(x))$, is considered to be given. Current continuity can then be expressed as (Lyons, 1980) 
Table 2. Populations and their associated piecewise linear current-voltage relation. Terms in black correspond to the simple model. A more realistic model includes also the red terms to account for zero-potential currents due to precipitation of hot plasma sheet particles and the outflow of heated ionospheric particles. Subscripts $m$ and i refer to magnetospheric and ionospheric particles, respectively, while + and indicate ions and electrons.

\begin{tabular}{|c|c|c|c|c|}
\hline population & & & $\Delta_{\|} \phi<0$ & $\Delta_{\|} \phi \geq 0$ \\
\hline lobe electrons & $j_{\mathrm{m}-}$ & $=$ & 0 & 0 \\
\hline lobe ions & $j_{\mathrm{m}+}$ & $=$ & 0 & 0 \\
\hline polar cap electrons & $j_{\mathrm{i}-}$ & $=$ & $+K_{\mathrm{i}-} \Delta_{\|} \phi$ & 0 \\
\hline polar cap ions & $j_{i+}$ & $=$ & $-K_{\mathrm{i}+} \Delta_{\|} \phi$ & 0 \\
\hline plasma sheet electrons & $j_{\mathrm{m}-}$ & $=$ & $j_{\mathrm{m}-0}+\tilde{K}_{\mathrm{m}-\Delta_{\|} \phi}$ & $j_{\mathrm{m}-0}+\tilde{K}_{\mathrm{m}-} \Delta_{\|} \phi+K_{\mathrm{m}-} \Delta_{\|} \phi$ \\
\hline plasma sheet ions & $j_{\mathrm{m}+}$ & $=$ & $j_{\mathrm{m}+0}-\tilde{K}_{\mathrm{m}+\Delta_{\|}} \phi+K_{\mathrm{m}+\Delta_{\|} \phi}$ & $j_{\mathrm{m}+0}-\tilde{K}_{\mathrm{m}+\Delta_{\|} \phi}$ \\
\hline auroral oval electrons & $j_{\mathrm{i}-}$ & $=$ & $j_{\mathrm{i}-0}+\tilde{K}_{\mathrm{i}-} \Delta_{\|} \phi+K_{\mathrm{i}-} \Delta_{\|} \phi$ & $j_{\mathrm{i}-0}+\tilde{K}_{\mathrm{i}-\Delta_{\|} \phi} \phi$ \\
\hline auroral oval ions & $j_{\mathrm{i}+}$ & $=$ & $j_{\mathrm{i}+0}-\tilde{K}_{\mathrm{i}+} \Delta_{\|} \phi-K_{\mathrm{i}+} \Delta_{\|} \phi$ & $j_{\mathrm{i}+0}-\tilde{K}_{\mathrm{i}+} \Delta_{\|} \phi$ \\
\hline
\end{tabular}

$\frac{\mathrm{d}}{\mathrm{d} x}\left(\Sigma_{\mathrm{P}} \frac{\mathrm{d}}{\mathrm{d} x} \phi\right)=j_{\|}$,

where $\phi(x)$ is the ionospheric electric potential profile, $\Sigma_{\mathrm{P}}$ represents the height-integrated Pedersen conductivity, and $j_{\|}$is the field-aligned current density in or out of the ionosphere. The parallel currents $j_{\|}$are taken to be vertical, and positive for upward currents. This equation states that the divergence of $I_{\mathrm{P}}=-\Sigma_{\mathrm{P}} \mathrm{d} \phi / \mathrm{d} x$, the horizontal Pedersen current integrated over the ionosphere up to altitude $h$, is balanced by the field-aligned currents. The ionosphere acts as a load in the auroral current system, characterized by the Pedersen conductivity; this conductivity is taken constant here. Note that the Hall conductivity does not appear in the model, because the Hall currents do not contribute to current closure in the one-dimensional setup.

A crucial element in the auroral current system are the field-aligned currents. The current-voltage relation indicates how such currents relate to the field-aligned electric potential difference $\Delta_{\|} \phi=\phi-\hat{\phi}$ between the ionospheric $(\phi)$ and magnetospheric $(\hat{\phi})$ electric potentials. The present study employs a phenomenological approach by considering (piecewise) linear current-voltage relations (as in De Keyser and Echim, 2010). The fundamental principle is that the field-aligned current grows with $\Delta_{\|} \phi$ for particles of one sign, while particles of the opposite sign experience an electric potential barrier. We use two different models, both of which are summarized in Table 2 . The basic model includes only the black terms given in the table. For $\Delta_{\|} \phi<0$, a return current is the net result of upgoing ionospheric ions and electrons. Electrons are accelerated out of the ionosphere and some of the ions can escape too if they have enough energy to overcome the electric potential barrier, while the plasma tries to maintain charge neutrality. The analysis by Vedin and Rönnmark (2005) indicates that a substantial upward electron flux can indeed exist when the accelerating potential is at lower altitude; this is supported by observations of black aurora (Marklund et al., 2001). For $\Delta_{\|} \phi>0$ only precipitating plasma sheet electrons are considered as they usually are the main current carriers. A more advanced model, which incorporates also the red terms in the table, accounts for the precipitation and outflow offset currents $j_{\mathrm{PO}}$ that exist for $\Delta_{\|} \phi=0$ due to the hot plasma sheet electrons and the ions that precipitate via the loss cone, as well as due to the outflow of ionospheric ions and electrons in the auroral oval when it is bombarded by the hot plasma sheet particles. The constants in the current-voltage relation (the Knight constants and the zero-potential offsets) depend on source population properties, such as density and temperature, as well as on the geometric mapping between magnetosphere and ionosphere. The currents implied by the additional terms must be small; if not, they would lead to a build-up of a magnetosphereionosphere charge imbalance. Despite the fact that they are small, they may alter the configuration and affect the escape of ionospheric ions. Adding the current contributions of all populations leads to a combined current-voltage relation that is piecewise linear as well. The linear approximation has been used in the past (e.g. Lyons, 1980; Temerin and Carlson, 1998), but it obviously is a simplification. The individual currents are not independent from one another as charge neutrality must be maintained, and that would translate in a certain relationship between the different Knight constants, as well as a possible deviation from linearity. Nevertheless, the linear approximation can already capture a number of interesting properties of the actual physical system (e.g., De Keyser and Echim, 2010).

A monopolar electric field produces a step-like change $\Delta_{\perp} \hat{\phi}$ in the magnetospheric electric potential. We consider configurations as depicted in Fig. 3 where a transverse $\Delta_{\perp} \hat{\phi}$ exists across the lobe-plasma sheet boundary. This boundary maps onto the polar cap boundary, at the poleward edge of the auroral oval. The transverse potential difference drives field-aligned currents that close horizontally as a Pedersen current in the ionosphere; the upward currents are mainly due to precipitating electrons that produce auroral emission. The magnitude and sign of $\Delta_{\perp} \hat{\phi}$ depend on the overall state of the magnetosphere. The magnetospheric electric potential 


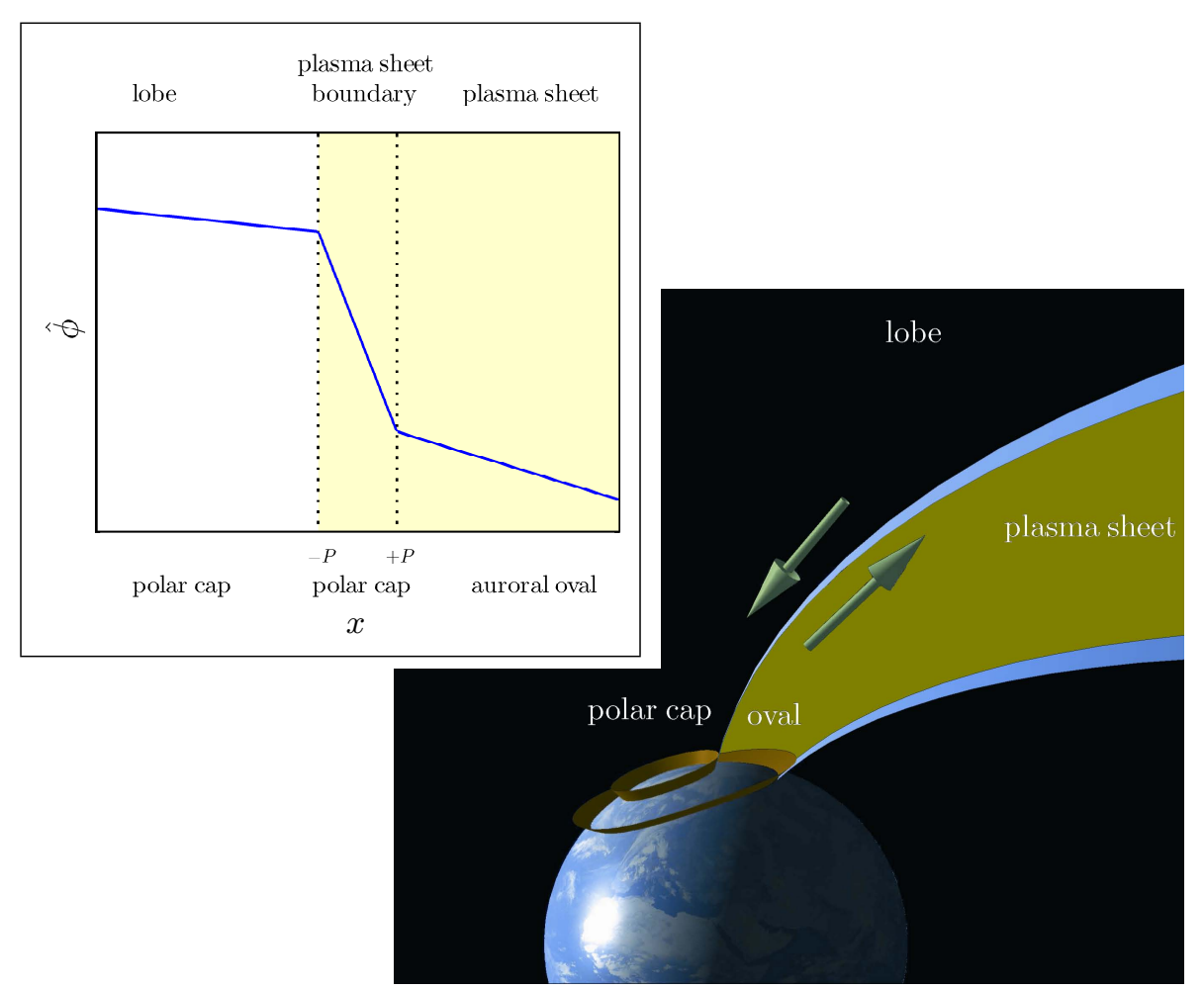

Fig. 3. Sketch of the geometry for monopolar electric fields at the polar cap boundary. The polar cap boundary separates the polar cap from the auroral oval and maps to the plasma sheet boundary layer, the interface between the lobe (black) and the plasma sheet (green). Up- and downward field-aligned currents (represented by arrows) flow on either side of the interface. The inset shows the one-dimensional situation considered here. The blue curve gives the magnetospheric electric potential $\hat{\phi}$ as a function of position across the structure, from pole to equator. The shaded region indicates where hot ion and electron precipitation and ionospheric ion outflow occur. Hot ion and electron precipitation extend throughout the interface.

distribution is closely related to plasma convection and reflects the solar wind-magnetosphere and the magnetosphereionosphere coupling. Typically, velocity shears may be responsible for the potential difference across a plasma interface. The sense of the velocity shear determines the sign of $\Delta_{\perp} \hat{\phi}$, which turns out to be a crucial parameter for the structure of the auroral current system.

\subsection{Monopolar field with $\Delta_{\perp} \hat{\phi}<0$}

Consider an interface with thickness $2 P$ that separates the cold lobe plasma on the left from the hot plasma sheet on the right, as in Fig. 3. The problem domain then consists of the lobe (above the polar cap, $x<-P$ ), the lobe-plasma sheet interface (corresponding to the polar cap boundary, $-P \leq$ $x \leq+P$ ), and the plasma sheet (above the auroral oval, $x>$ $P)$. The magnetospheric electric potential is represented by

$\hat{\phi}= \begin{cases}-\frac{1}{2} \Delta_{\perp} \hat{\phi}-\epsilon_{\mathrm{L}}(x+P), & x<-P, \\ \frac{1}{2} \Delta_{\perp} \hat{\phi} \frac{x}{P}, & -P \leq x \leq+P, \\ +\frac{1}{2} \Delta_{\perp} \hat{\phi}-\epsilon_{\mathrm{R}}(x-P), & +P \leq x .\end{cases}$
The monopolar magnetospheric electric field is taken constant inside the interface, $E_{x}=-\Delta_{\perp} \hat{\phi} / 2 P$. This is only an approximation as observations indicate a more smoothly varying electric field profile (Johansson et al., 2006). The electric fields $\epsilon_{\mathrm{L}}$ and $\epsilon_{\mathrm{R}}$ on either side of the lobe-plasma sheet interface are due to plasma convection. They are considered here to be relatively small.

Note that the thickness of the boundary is determined by the hot plasma sheet particle gyroradii. These hot particles are present throughout the transition, although their number actually depends on the sign of $\Delta_{\perp} \hat{\phi}$ (as evident in Fig. 1). That would, strictly speaking, affect the value of the corresponding Knight constant; such changes are not considered here.

\subsubsection{Basic field-aligned current model}

If $\epsilon_{\mathrm{L}}$ and $\epsilon_{\mathrm{R}}$ are small, there clearly must be a point $\xi \epsilon$ $[-P,+P]$ inside the transition where $\Delta_{\|} \phi$ changes sign. The basic field-aligned current model can then be summarized in a combined current-voltage relation above the auroral oval and the equatorward part of the boundary, where $\Delta_{\|} \phi \geq 0$, 
as

$j_{\|}=K_{\mathrm{m}-} \Delta_{\|} \phi=K_{+} \Delta_{\|} \phi$.

Above the poleward part of the boundary, where $\Delta_{\|} \phi<0$, the current-voltage relation is

$j_{\|}=\left(K_{\mathrm{i}-}-K_{\mathrm{i}+}+K_{\mathrm{m}+}\right) \Delta_{\|} \phi=K_{*} \Delta_{\|} \phi$,

while above the polar cap, where $\Delta_{\|} \phi<0$ and where there is no precipitation,

$j_{\|}=\left(K_{\mathrm{i}-}-K_{\mathrm{i}+}\right) \Delta_{\|} \phi=K_{-} \Delta_{\|} \phi$.

$K_{+}, K_{*}$, and $K_{-}$are the overall Knight constants, with $K_{*} \geq K_{-}$. Armed with these current-voltage relations, current continuity can be expressed as

$\frac{\mathrm{d}^{2} \phi}{\mathrm{d} x^{2}}= \begin{cases}\frac{1}{\lambda_{-}^{2}}(\phi-\hat{\phi}), & x<-P, \\ \frac{1}{\lambda_{*}^{2}}(\phi-\hat{\phi}), & -P \leq x<\xi, \\ \frac{1}{\lambda_{+}^{2}}(\phi-\hat{\phi}), & \xi \leq x,\end{cases}$

where $\quad \lambda_{+}=\sqrt{\Sigma_{\mathrm{P}} / K_{+}}, \quad \lambda_{*}=\sqrt{\Sigma_{\mathrm{P}} / K_{*}}$ and $\lambda_{-}=$ $\sqrt{\Sigma_{\mathrm{P}} / K_{-}} \geq \lambda_{*}$ define the characteristic length scales. Writing down the form of the solutions in the four regions ]$-\infty,-P[,[-P, \xi[,[\xi,+P[$, and $[+P,+\infty[$, each having a linear magnetospheric potential profile and being characterized by a specific Knight constant, and imposing the boundary conditions $j_{\|}( \pm \infty)=0$ and the continuity conditions for $\phi(x)$ and $I_{\mathrm{P}}(x)$ at $-P, \xi$, and $+P$, leads to a linear system of 8 equations in 8 coefficients. This allows the problem to be solved in terms of $\xi$, which is determined from $\Delta_{\|} \phi(\xi)=0$. The full analytical solution is given in Appendix A. To gain more insight, we look at a few special cases.

When the current carried by precipitating magnetospheric ions in the return current region is negligible relative to the current carried by the upgoing ionospheric particles $\left(K_{\mathrm{i}-}-\right.$ $K_{\mathrm{i}+} \gg K_{\mathrm{m}+}$ ), then $K_{*}=K_{-}$and $\lambda_{*}=\lambda_{-}$. The point separating the up- and downward current regions can then be found explicitly as

$\xi=\frac{\lambda_{-}-\lambda_{+}}{\lambda_{-}+\lambda_{+}} P-\frac{\lambda_{-} \lambda_{+}}{\lambda_{-}+\lambda_{+}} \log \frac{\Delta_{\perp} \hat{\phi}+2 \epsilon_{\mathrm{R}} P}{\Delta_{\perp} \hat{\phi}+2 \epsilon_{\mathrm{L}} P}$

as long as the convection electric fields are small and/or the transition is sufficiently thin, so that the potential difference across the structure dominates and the argument of the logarithm is positive. The corresponding ionospheric potential is

$\phi=\hat{\phi}+\left\{\begin{array}{r}\frac{\left(\Delta_{\perp} \hat{\phi}+2 \epsilon_{\mathrm{L}} P\right) \lambda_{-}}{4 P}\left(1-\mathrm{e}^{-2(\xi+P) / \lambda_{-}}\right) \mathrm{e}^{(x+P) / \lambda_{-}}, \\ x<-P, \\ \frac{\left(\Delta_{\perp} \hat{\phi}+2 \epsilon_{\mathrm{L}} P\right) \lambda_{-}}{4 P} \mathrm{e}^{-P / \lambda_{-}}\left(\mathrm{e}^{-x / \lambda_{-}}-\mathrm{e}^{(x-2 \xi) / \lambda_{-}}\right), \\ -P \leq x<\xi, \\ \frac{\left(\Delta_{\perp} \hat{\phi}+2 \epsilon_{\mathrm{R}} P\right) \lambda_{+}}{4 P} \mathrm{e}^{-P / \lambda_{+}}\left(\mathrm{e}^{\left.-(x-2 \xi) / \lambda_{+}-\mathrm{e}^{x / \lambda_{+}}\right),}\right. \\ \xi \leq x<+P, \\ \frac{\left(\Delta_{\perp} \hat{\phi}+2 \epsilon_{\mathrm{R}} P\right) \lambda_{+}}{4 P}\left(-1+\mathrm{e}^{2(\xi-P) / \lambda_{+}}\right) \mathrm{e}^{-(x-P) / \lambda_{+}}, \\ +P \leq x .\end{array}\right.$
This solution exhibits a left/right symmetry, i.e., it is invariant for a simultaneous exchange of the variables $\lambda_{-} \leftrightarrow \lambda_{+}$, $-x \leftrightarrow+x,-\epsilon_{\mathrm{L}} \leftrightarrow+\epsilon_{\mathrm{R}}$, and $-\Delta_{\perp} \hat{\phi} \leftrightarrow+\Delta_{\perp} \hat{\phi}$. (Note that $\xi$ also changes sign for such an exchange.)

A second special case occurs when the return current is rather strong. A strong return current can arise as a negative parallel potential difference might be able to produce a fairly rapid upward acceleration of the electrons and therefore a local depletion of the ionospheric electrons. This would have two consequences: a large Knight constant $\left(K_{-} \gg K_{+}\right)$and a low conductivity (an effect that we do not model here). Both lead to $\lambda_{*} \leq \lambda_{-} \ll P$, while $\lambda_{+}$is on the order of $P$. One then finds that $\xi=-P$, and the solution is

$\phi=\hat{\phi}-\left\{\begin{array}{cc}0, & x<-P, \\ \frac{\left(\Delta_{\perp} \hat{\phi}+2 \epsilon_{\mathrm{R}} P\right) \lambda_{+}}{2 P} \mathrm{e}^{-2 P / \lambda_{+}} \sinh \frac{x+P}{\lambda_{+}}, & -P \leq x<+P, \\ \frac{\left(\Delta_{\perp} \hat{\phi}+2 \epsilon_{\mathrm{R}} P\right) \lambda_{+}}{4 P}\left(1-\mathrm{e}^{-2 P / \lambda_{+}}\right) & \mathrm{e}^{-(x-P) / \lambda_{+},} \\ +P \leq x,\end{array}\right.$

in which there is no parallel potential difference for $x<-P$. Note that $\lambda_{*}$ and $\lambda_{-}$have disappeared from the solution.

A final special case is the thin interface limit, when $P \ll$ $\lambda_{*} \leq \lambda_{-}$and $P \ll \lambda_{+}$, i.e., when the characteristic length scales associated with the auroral circuit are all larger than the interface thickness. The condition $\Delta_{\|} \phi(\xi)=0$ then amounts to

$\xi=\frac{P}{\lambda_{-}+\lambda_{+}}\left[\left(\lambda_{-}-\lambda_{+}\right)-\frac{2 \lambda_{-} \lambda_{+} \Delta \epsilon}{\Delta_{\perp} \hat{\phi}}\right]$,

where $\Delta \epsilon=\epsilon_{\mathrm{R}}-\epsilon_{\mathrm{L}}$, so that $\xi \rightarrow 0$ as $P \rightarrow 0$. Note that $\lambda_{*}$ plays no role as the region with magnetospheric ion precipitation becomes vanishingly thin. The ionospheric potential is

$\phi=\hat{\phi}+ \begin{cases}\left(\Delta_{\perp} \hat{\phi}-\lambda_{+} \Delta \epsilon\right) \frac{\lambda_{-} \mathrm{e}^{x / \lambda_{-}}}{\lambda_{-}+\lambda_{+}}, & x<0, \\ -\left(\Delta_{\perp} \hat{\phi}+\lambda_{-} \Delta \epsilon\right) \frac{\lambda_{+} \mathrm{e}^{-x / \lambda_{+}}}{\lambda_{-}+\lambda_{+}}, & 0 \leq x,\end{cases}$

This solution has the same left/right symmetry discussed before: It is invariant for an exchange of the variables $\lambda_{-} \leftrightarrow \lambda_{+}$, $-x \leftrightarrow+x,-\epsilon_{\mathrm{L}} \leftrightarrow+\epsilon_{\mathrm{R}}$, and $-\Delta_{\perp} \hat{\phi} \leftrightarrow+\Delta_{\perp} \hat{\phi}$. The corresponding field-aligned currents are

$j_{\|}= \begin{cases}K_{-}\left(\Delta_{\perp} \hat{\phi}-\lambda_{+} \Delta \epsilon\right) \frac{\lambda_{-} \mathrm{e}^{x / \lambda_{-}}}{\lambda_{-}+\lambda_{+}}, & x<0, \\ -K_{+}\left(\Delta_{\perp} \hat{\phi}+\lambda_{-} \Delta \epsilon\right) \frac{\lambda_{+} \mathrm{e}^{-x / \lambda_{+}}}{\lambda_{-}+\lambda_{+}}, & 0 \leq x,\end{cases}$

The current forms two current sheets carrying upward/downward field-aligned currents on either side of the lobe-plasma sheet interface, with thicknesses $\lambda_{-}$and $\lambda_{+}$. It is interesting to compute the total parallel current on either 
side of the interface. These integrated currents are

$$
\begin{aligned}
& J_{\downarrow}=\int_{-\infty}^{0} j_{\|}(x) \mathrm{d} x=\Sigma_{\mathrm{P}} \frac{\Delta_{\perp} \hat{\phi}-\lambda_{+} \Delta \epsilon}{\lambda_{-}+\lambda_{+}}, \\
& J_{\uparrow}=\int_{0}^{+\infty} j_{\|}(x) \mathrm{d} x=-\Sigma_{\mathrm{P}} \frac{\Delta_{\perp} \hat{\phi}+\lambda_{-} \Delta \epsilon}{\lambda_{-}+\lambda_{+}} .
\end{aligned}
$$

The two currents are exactly opposite when the convection electric field difference is small, $|\Delta \epsilon| \ll$ $\left|\Delta_{\perp} \hat{\phi}\right| / \max \left\{\lambda_{-}, \lambda_{+}\right\}$, so that there is a local closure of the currents flowing between the magnetosphere and the ionosphere.

Combining the thin interface approximation with the strong return current limit, a marked asymmetry between the two sides arises as $\lambda_{-} \ll \lambda_{+}$(when $\left.K_{-} \gg K_{+}\right)$. As the scale length $\lambda_{-}$decreases, the return current region becomes narrower while the peak return current increases, so as to maintain the balance between $J_{\downarrow}$ and $J_{\uparrow}$. The solution is

$\phi=\hat{\phi}- \begin{cases}0, & x<0, \\ \Delta_{\perp} \hat{\phi} \mathrm{e}^{-x / \lambda_{+},} & 0 \leq x .\end{cases}$

The field-aligned currents are

$j_{\|}= \begin{cases}K_{-}\left(\Delta_{\perp} \hat{\phi}-\lambda_{+} \Delta \epsilon\right) \frac{\lambda_{-}}{\lambda_{+}} \delta\left(\frac{x}{\lambda_{-}}\right), & x<0, \\ -K_{+} \Delta_{\perp} \hat{\phi} \mathrm{e}^{-x / \lambda_{+}}, & 0 \leq x,\end{cases}$

where $\delta(x)$ is the Dirac function representing a thin current sheet on the left side. The integrated currents are

$J_{\downarrow}=\Sigma_{\mathrm{P}} \frac{\Delta_{\perp} \hat{\phi}-\lambda_{+} \Delta \epsilon}{\lambda_{+}}, \quad J_{\uparrow}=-\Sigma_{\mathrm{P}} \frac{\Delta_{\perp} \hat{\phi}}{\lambda_{+}}$,

which balance each other when the convection electric fields are small.

Figure 4 shows a typical lobe-plasma sheet boundary configuration, with an electric potential jump $\Delta_{\perp} \hat{\phi}=-5 \mathrm{kV}$ and convection electric fields $\epsilon_{\mathrm{L}}=10 \mathrm{mV} / \mathrm{m}$ and $\epsilon_{\mathrm{R}}=30 \mathrm{mV} / \mathrm{m}$ (panel a). All quantities are plotted as a function of $x$, the transverse horizontal coordinate at the altitude of the topside ionosphere. The magnetic field mapping factor is typically $b=\hat{x}(x) / x \approx 10$ if the topside ionosphere is at $h=400 \mathrm{~km}$ and if the magnetospheric altitude is $5 R_{\mathrm{E}}$. These parameters are such that they reproduce the observed parallel current densities at ionospheric altitude on the order of $1-10 \mu \mathrm{A} / \mathrm{m}^{2}$ for accelerating potentials $\Delta_{\|} \phi$ on the order of a kiloVolt, while the parallel current densities observed at high altitude are a factor $b^{2}$ smaller, i.e., two orders of magnitude lower (Figueiredo et al., 2005; Johansson et al., 2005, 2006, 2007). The interface has a half-thickness $P=5 \mathrm{~km}$ in the ionosphere, typical of observed discrete auroral arc curtains and corresponding to a half-thickness $b P=50 \mathrm{~km}$ in the magnetosphere. Note also that $P<\lambda_{+}$and $P<\lambda_{-}$. If $P$ is larger than both, one deals with a pair of converging and diverging electric field configurations so far apart that they can be

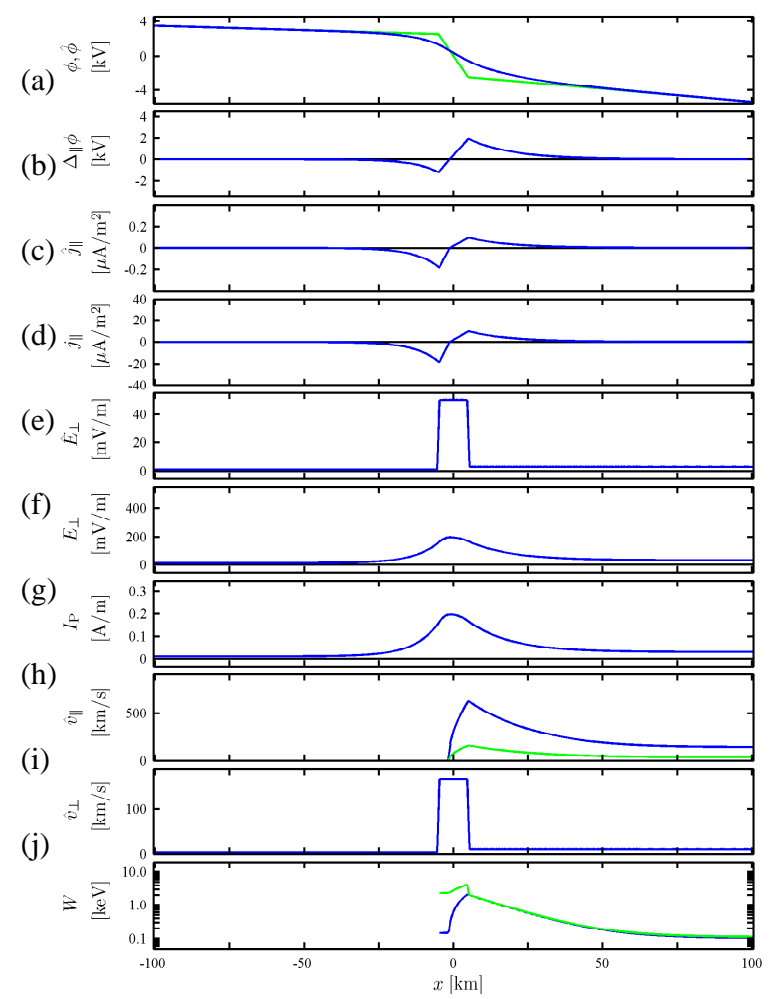

Fig. 4. Auroral current structure for a monopolar electric field at the lobe-plasma sheet boundary, with an electric potential jump $\Delta_{\perp} \hat{\phi}=-5 \mathrm{kV}$ and convection electric fields $\epsilon_{\mathrm{L}}=10 \mathrm{mV} / \mathrm{m}$ and $\epsilon_{\mathrm{R}}=30 \mathrm{mV} / \mathrm{m}$. The magnetic field mapping factor used to reproduce magnetospheric quantities is $b=10$, corresponding to a magnetospheric altitude just above the parallel acceleration region. The boundary thickness is $10 \mathrm{~km}$ at ionospheric altitude $(100 \mathrm{~km}$ at magnetospheric altitude). All quantities are plotted as a function of $x$, the transverse horizontal coordinate at the altitude of the topside ionosphere. The height-integrated Pedersen conductivity is $1 \mathrm{~S}$. The basic field-aligned current model was used, with Knight constants $K_{-}=15 \mu \mathrm{A} / \mathrm{kV} \mathrm{m}^{2}, K_{*}=17 \mu \mathrm{A} / \mathrm{kV} \mathrm{m}^{2}$, and $K_{+}=5 \mu \mathrm{A} / \mathrm{kV} \mathrm{m}^{2}$. (a) Ionospheric and magnetospheric potential, blue resp. green; (b) Parallel potential difference; (c) Field-aligned current at magnetospheric and (d) at ionospheric altitude; (e) Perpendicular electric field at magnetospheric and (f) ionospheric altitude; (g) Heightintegrated Pedersen current; (h) Parallel and (i) perpendicular velocity at magnetospheric altitude, and (j) energy of upgoing ions that escape from the ionosphere with an initial energy $W_{0}=100 \mathrm{eV}$ $\left(\mathrm{H}^{+}\right.$, blue, and $\mathrm{O}^{+}$, green).

treated separately (De Keyser and Echim, 2010). The electric potential jump $\Delta_{\perp} \hat{\phi}=-5 \mathrm{kV}$ implies a monopolar field of $50 \mathrm{mV} / \mathrm{m}$ at magnetospheric altitude.

The ionospheric potential (panel a, blue) is a "smoothed" version of the magnetospheric profile (panel a, green). The field-aligned potential difference $\Delta_{\|} \phi$ is indeed $<0$ on the lobe side and $>0$ on the plasma sheet side (panel b), with $\Delta_{\|} \phi$ changing sign at $\xi=-1.2 \mathrm{~km}$. The potential difference peaks at -1.2 and $+1.9 \mathrm{kV}$. The return current is located poleward of the interface, while the upward current region is 
found on the equatorward side (panels $\mathrm{c}$ and $\mathrm{d}$ ). The width of the parallel current regions is of the order of $\lambda_{-}=8.2 \mathrm{~km}$ and $\lambda_{+}=14.1 \mathrm{~km}$, respectively $\left(\lambda_{*}=7.7 \mathrm{~km}\right.$ plays only a secondary role inside the return current region). The narrower return current region on the polar cap side has more intense currents than the broader upward current on the side of the oval, since the total up- and downward currents must balance. While the monopolar magnetospheric electric field perpendicular to the lobe-plasma sheet interface has the rectangular shape implicit in the imposed magnetospheric potential (panel e), the ionospheric electric field transverse to the auroral structure is also monopolar but more smoothly varying (panel f). The height-integrated Pedersen current (panel g) peaks near the center of the polar cap boundary.

It is particularly instructive to examine what happens in the auroral oval. Plasma sheet electrons are accelerated downward and precipitate. However, since the hot plasma sheet ions have an energy $W_{\perp+}^{\prime}$ in $\mathrm{keV}$ that is larger than $\Delta_{\perp} \hat{\phi}$ in $\mathrm{kV}$ (inequality 2 derived above), the plasma sheet ions can precipitate as well, despite some parallel deceleration, since $\Delta_{\|} \phi<\Delta_{\perp} \hat{\phi}<2 \ell_{+} W_{\perp+}^{\prime} / Z_{+} \approx 2 W_{\perp+}^{\prime}$. The ions in the upper ionosphere gain energy through the mechanism of transverse ion heating by the lower hybrid waves excited by the precipitation (Eliasson et al., 1994; Moore et al., 1999). This may lead to an outflow of the heated ions, mostly protons but with a significant admixture of oxygen ions. During outflow in the diverging magnetic field, the perpendicular energy of these particles is converted adiabatically into parallel energy. Take this initial energy to be $W_{0}=100 \mathrm{eV}$, a realistic value (Eliasson et al., 1994). The assumption of a constant initial energy is of course quite crude. In reality it depends on the ion mass, the ionospheric conditions, and the precipitation, but as the ionospheric ions are used here as "tracers" to illustrate the basic behaviour, this assumption is sufficient. In addition to the initial escape velocity corresponding to $W_{0}$, the ionospheric ions experience a further parallel acceleration by the parallel potential difference as they flow upward, leading to significant parallel velocities at high altitude (panel $\mathrm{h}$, blue for $\mathrm{H}^{+}$ and green for $\mathrm{O}^{+}$). The parallel velocity of the $\mathrm{O}^{+}$ions is about 1/4th that of the protons because the energy gain is mass-independent. At the same time, these ions experience an $\boldsymbol{E} \times \boldsymbol{B}$ drift, which gives them a perpendicular velocity $v_{\perp}=E_{\perp} / B$ (panel i). This produces a peculiar behaviour of the upgoing ion energies (panel $\mathrm{j}$ ). Inside the transition, the $\mathrm{O}^{+}$energy exceeds that of $\mathrm{H}^{+}$: the perpendicular drift speed increases monotonically with altitude in a mass-independent manner, so that the $\mathrm{O}^{+}$ions acquire 4 times more perpendicular energy than the protons. Outside the transition, the energies of $\mathrm{H}^{+}$and $\mathrm{O}^{+}$are identical as $W_{0}$ was assumed to be the same for both species and as they are accelerated through the same potential difference. The outflowing ions have a high energy inside the boundary, which drops off on a length scale $\lambda_{+}$towards the background energy $W_{0}$. This result can also be interpreted in terms of the particle pitch angles: inside the transition the ionospheric ions have mainly perpendicular energy (pitch angle $90^{\circ}$ ), while they have an essentially parallel motion above the auroral oval (pitch angle $0^{\circ}$ or $180^{\circ}$ depending on the hemisphere).

\subsubsection{Advanced field-aligned current model}

Consider now the same problem, but with the more advanced field-aligned current model that includes (small) precipitation/outflow offset currents. Such currents are only present above the oval and its boundary, not above the polar cap. The current-voltage relation there is, for $\Delta_{\|} \phi \geq 0$, (slightly) modified to

$$
\begin{aligned}
j_{\|}= & \left(j_{\mathrm{m}-0}+j_{\mathrm{m}+0}+j_{\mathrm{i}-0}+j_{\mathrm{i}+0}\right) \\
& +\left(K_{\mathrm{m}-}+\tilde{K}_{\mathrm{m}-}-\tilde{K}_{\mathrm{m}+}+\tilde{K}_{\mathrm{i}--}-\tilde{K}_{\mathrm{i}+}\right) \Delta_{\|} \phi \\
= & j_{0}+\left(K_{+}+K_{0}\right) \Delta_{\|} \phi=j_{\mathrm{PO}}+K_{+} \Delta_{\|} \phi,
\end{aligned}
$$

with precipitation/outflow offset current $j_{\mathrm{PO}}=j_{0}+K_{0} \Delta_{\|} \phi$, where $j_{0}=j_{\mathrm{m}-0}+j_{\mathrm{m}+0}+j_{\mathrm{i}-0}+j_{\mathrm{i}+0}$ and $K_{0}=\tilde{K}_{\mathrm{m}-}-$ $\tilde{K}_{\mathrm{m}+}+\tilde{K}_{\mathrm{i}-}-\tilde{K}_{\mathrm{i}+}$. For $\Delta_{\|} \phi<0$, the current-voltage relation there now is

$$
\begin{aligned}
j_{\|}= & \left(j_{\mathrm{m}-0}+j_{\mathrm{m}+0}+j_{\mathrm{i}-0}+j_{\mathrm{i}+0}\right) \\
& +\left(K_{\mathrm{m}+}+K_{\mathrm{i}-}-K_{\mathrm{i}+}\right. \\
& \left.\quad+\tilde{K}_{\mathrm{m}-}-\tilde{K}_{\mathrm{m}+}+\tilde{K}_{\mathrm{i}-}-\tilde{K}_{\mathrm{i}+}\right) \Delta_{\|} \phi \\
= & j_{0}+\left(K_{*}+K_{0}\right) \Delta_{\|} \phi=j_{\mathrm{PO}}+K_{*} \Delta_{\|} \phi .
\end{aligned}
$$

Above the polar cap, where $\Delta_{\|} \phi<0$, the relation remains

$j_{\|}=\left(K_{\mathrm{i}-}-K_{\mathrm{i}+}\right) \Delta_{\|} \phi=K_{-} \Delta_{\|} \phi$.

As long as $K_{0}$ is small compared to $K_{-}, K_{*}$, and $K_{-}$, the $\Delta_{\|} \phi$-dependence of $j_{\|}$doesn't change much. The presence of a small $j_{0}$, however, has a fundamental effect on the nature of the solution. One must distinguish two different situations.

\section{Negative precipitation/outflow offset current}

The boundary condition $j_{\|}(+\infty)=0$ requires that $\Delta_{\|} \phi(+\infty)>0$ when $j_{\mathrm{PO}}<0$. The overall configuration then is the same as before, with a return current on the poleward (left) side and an upward current on the equatorward (right) side of the interface. Current continuity implies

$\frac{\mathrm{d}^{2} \phi}{\mathrm{d} x^{2}}= \begin{cases}\frac{1}{\lambda_{-}^{2}}(\phi-\hat{\phi}), & x<-P, \\ \frac{1}{\tilde{\lambda}_{*}^{2}}\left(\phi-\hat{\phi}+\Delta \phi_{*}\right), & -P \leq x<\xi, \\ \frac{1}{\tilde{\lambda}_{+}^{2}}\left(\phi-\hat{\phi}+\Delta \phi_{+}\right), & \xi \leq x,\end{cases}$

where $\tilde{\lambda}_{*}=\sqrt{\Sigma_{\mathrm{P}} /\left(K_{*}+K_{0}\right)}, \quad \tilde{\lambda}_{+}=\sqrt{\Sigma_{\mathrm{P}} /\left(K_{+}+K_{0}\right)}$, $\Delta \phi_{*}=j_{0} /\left(K_{*}+K_{0}\right)$, and $\Delta \phi_{+}=j_{0} /\left(K_{+}+K_{0}\right)$. One may regard $\Delta \phi_{*}$ and $\Delta \phi_{+}$as equivalent potentials that would produce a parallel current $j_{0}$ with the given Knight constants $K_{*}$ and $K_{+}$, respectively.

Expressing the boundary conditions and the continuity of $\phi(x)$ and $I_{\mathrm{P}}(x)$ at $-P, \xi$, and $+P$ again gives a linear $8 \times 8$ 


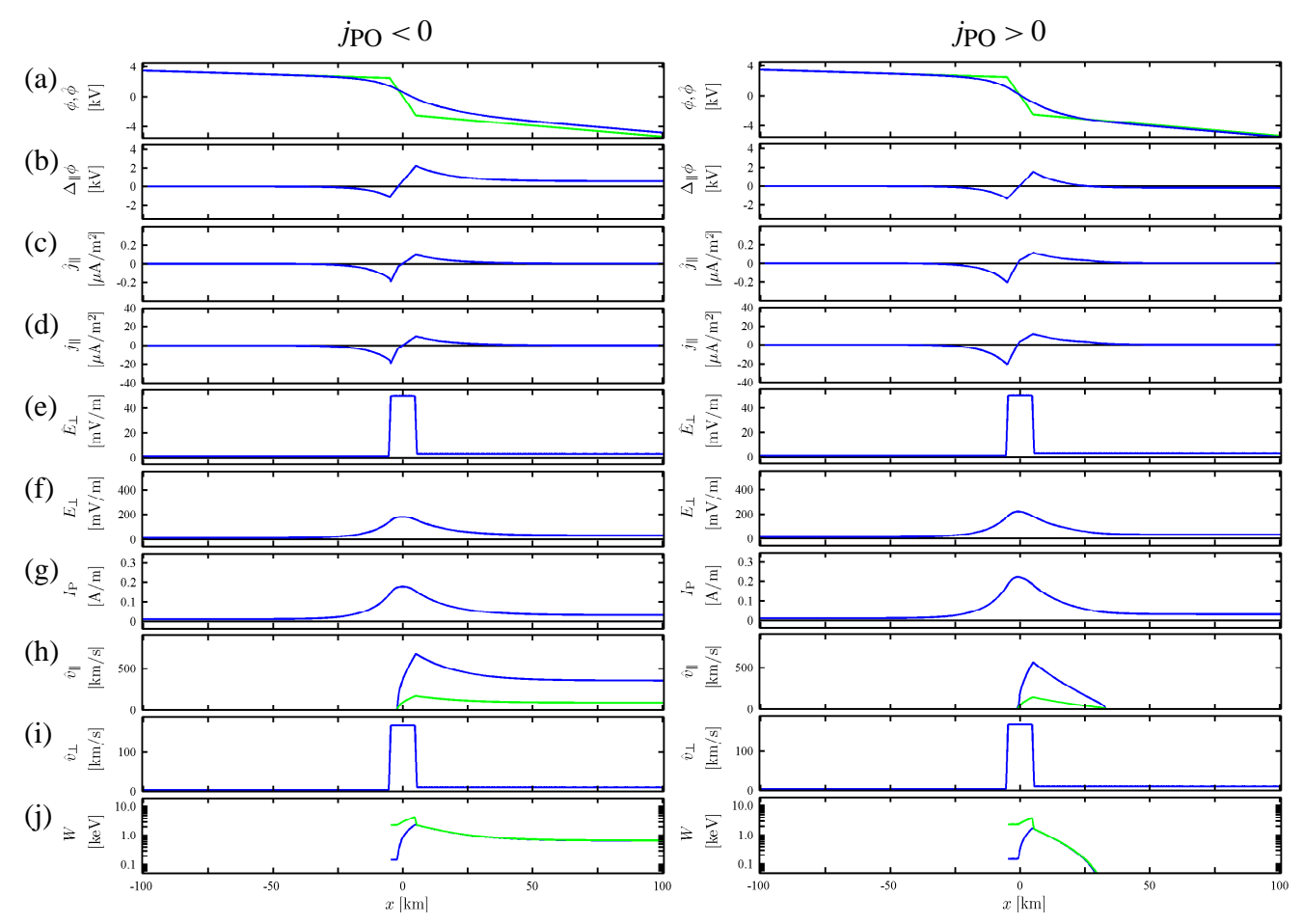

Fig. 5. Auroral current structure for a monopolar electric field at the lobe-plasma sheet boundary, similar to Fig. 4, but now computed with the advanced field-aligned current model. Left: $j_{0}=-3 \mu \mathrm{A} / \mathrm{m}^{2}$ and $K_{0}=0.5 \mu \mathrm{A} / \mathrm{kV} \mathrm{m}{ }^{2}$. Right: $j_{0}=+3 \mu \mathrm{A} / \mathrm{m}^{2}$ and $K_{0}=0.5 \mu \mathrm{A} / \mathrm{kV} \mathrm{m}^{2}$.

system, leading to a solution in terms of $\xi$, the point where $\Delta_{\|} \phi$ changes sign inside the transition. The analytical expressions are too complicated to be given here, but the problem can also be solved numerically.

It is instructive to take a look at the thin interface limit, $P \rightarrow 0$. In that case $\xi \rightarrow 0$. The solution then is

$\phi=\hat{\phi}+\left\{\begin{array}{lr}\left(\Delta_{\perp} \hat{\phi}-\Delta \phi_{+}-\tilde{\lambda}_{+} \Delta \epsilon\right) \frac{\lambda_{-} \mathrm{e}^{x / \lambda_{-}}}{\lambda_{-}+\tilde{\lambda}_{+}}, & x<0, \\ -\left(\Delta_{\perp} \hat{\phi}-\Delta \phi_{+}+\lambda_{-} \Delta \epsilon\right) \frac{\tilde{\lambda}_{+} \mathrm{e}^{-x / \tilde{\lambda}_{+}}}{\lambda_{-}+\tilde{\lambda}_{+}}-\Delta \phi_{+}, & 0 \leq x,\end{array}\right.$

with the field-aligned currents

$j_{\|}=\left\{\begin{array}{lr}K_{-}\left(\Delta_{\perp} \hat{\phi}-\Delta \phi_{+}-\tilde{\lambda}_{+} \Delta \epsilon\right) \frac{\lambda_{-} \mathrm{e}^{x / \lambda_{-}}}{\lambda_{-}+\tilde{\lambda}_{+}}, & x<0, \\ -\left(K_{+}+K_{0}\right)\left(\Delta_{\perp} \hat{\phi}-\Delta \phi_{+}+\lambda_{-} \Delta \epsilon\right) \frac{\tilde{\lambda}_{+} \mathrm{e}^{-x / \tilde{\lambda}_{+}}}{\lambda_{-}+\tilde{\lambda}_{+}}, & 0 \leq x,\end{array}\right.$

from which $\tilde{\lambda}_{*}$ and $\Delta \phi_{*}$ have disappeared. Note that $\phi(+\infty)=-\Delta \phi_{+}>0$ and that $j_{\|}(+\infty)=0$ to satisfy the boundary conditions.

Figure 5, left column, shows the lobe-plasma sheet boundary configuration, for the same parameters as before. A small precipitation/outflow offset current is added, characterized by $j_{0}=-3 \mu \mathrm{A} / \mathrm{m}^{2}$ and $K_{0}=0.5 \mu \mathrm{A} / \mathrm{kVm}^{2}$, values that make sense when compared to the field-aligned currents produced by parallel acceleration. The ionospheric potential (panel a, blue) remains above the magnetospheric potential for $x \rightarrow+\infty$ (panel a, green), so that $\Delta_{\|} \phi<0$ on the lobe side and $\Delta_{\|} \phi>0$ on the plasma sheet side, changing sign at $\xi=-1.8 \mathrm{~km}$ (slightly poleward of its position in the absence of a precipitation/outflow offset current), and asymptotically reaching the value $\Delta \phi_{+}$(panel b). The presence of the small $j_{\mathrm{PO}}$ does not significantly affect the electrostatic structure: The field-aligned currents, the electric fields, and the Pedersen current remain essentially the same (panels c-g). What does change, however, are the properties of the outflowing ionospheric ions. Since there exists a nonzero positive $\Delta_{\|} \phi \geq \Delta \phi_{+}$throughout the auroral oval region, the upgoing ions experience parallel electrostatic acceleration throughout that region (panel h). The perpendicular ion velocities are not really affected (panel i). As a result, the energy of the outflowing ions above the oval is noticeably higher, at a value $W_{0}+Z_{+} e \Delta \phi_{+}$away from the interface (panel j).

\section{Positive precipitation/outflow offset current}

When $j_{\mathrm{PO}}>0$, the parallel current can vanish asymptotically only if $\Delta_{\|} \phi<0$ there. The configuration is now a bit more complicated: For a small positive $j_{\mathrm{PO}}>0$, the parallel potential difference is negative for $x<\xi$, with $\xi$ inside the transition, positive for $\xi \leq x \leq \eta$, with $\eta>P$, and negative again 
for $x>\eta$. Current continuity states that

$$
\frac{\mathrm{d}^{2} \phi}{\mathrm{d} x^{2}}= \begin{cases}\frac{1}{\lambda_{-}^{2}}(\phi-\hat{\phi}), & x<-P, \\ \frac{1}{\tilde{\lambda}_{*}^{2}}\left(\phi-\hat{\phi}+\Delta \phi_{*}\right), & -P \leq x<\xi, \\ \frac{1}{\tilde{\lambda}_{+}^{2}}\left(\phi-\hat{\phi}+\Delta \phi_{+}\right), & \xi \leq x<\eta, \\ \frac{1}{\tilde{\lambda}_{*}^{2}}\left(\phi-\hat{\phi}+\Delta \phi_{*}\right), & \eta \leq x .\end{cases}
$$

Expressing the boundary conditions and the continuity of $\phi$ and $I_{\mathrm{P}}$ at $x=-P, \xi,+P$, and $\eta$, results in a $10 \times 10$ linear system that gives the solution in terms of $\xi$ and $\eta$. These transition points are found from the nonlinear algebraic system $\Delta_{\|} \phi(\xi)=0$ and $\Delta_{\|} \phi(\eta)=0$, which can be solved numerically.

The problem is more tractable in the thin interface limit $P \rightarrow 0$, when $\xi=0$. Current continuity then is expressed by

$\frac{\mathrm{d}^{2} \phi}{\mathrm{d} x^{2}}= \begin{cases}\frac{1}{\lambda_{-}^{2}}(\phi-\hat{\phi}), & x<0, \\ \frac{1}{\tilde{\lambda}_{+}^{2}}\left(\phi-\hat{\phi}+\Delta \phi_{+}\right), & 0 \leq x<\eta, \\ \frac{1}{\tilde{\lambda}_{*}^{2}}\left(\phi-\hat{\phi}+\Delta \phi_{*}\right), & \eta \leq x,\end{cases}$

with the notation introduced before. Expressing the boundary conditions and the continuity of $\phi(x)$ and $I_{\mathrm{P}}(x)$ at $x=0$ and $x=\eta$, results in a $6 \times 6$ system that gives the solution in terms of $\eta$. The latter is found from the condition that $\Delta_{\|} \phi(\eta)=0$, which is the nonlinear algebraic equation

$$
\begin{aligned}
\left(\frac{\Delta \phi_{+}}{\tilde{\lambda}_{+}}+\right. & \left.\frac{\Delta \phi_{*}}{\tilde{\lambda}_{*}}\right)\left(\tilde{\lambda}_{+}+\lambda_{-}\right) \mathrm{e}^{2 \eta / \tilde{\lambda}_{+}} \\
& +2\left(\Delta_{\perp} \hat{\phi}-\Delta \phi_{+}+\lambda_{-} \Delta \epsilon\right) \mathrm{e}^{\eta / \tilde{\lambda}_{+}} \\
& +\left(\frac{\Delta \phi_{+}}{\tilde{\lambda}_{+}}-\frac{\Delta \phi_{*}}{\tilde{\lambda}_{*}}\right)\left(\tilde{\lambda}_{+}-\lambda_{-}\right)=0 .
\end{aligned}
$$

This equation is quadratic in $\mathrm{e}^{\eta / \tilde{\lambda}_{+}}$and can be solved analytically. An approximation is found by observing that $j_{\mathrm{PO}}$ is small, so that $\eta \gg \tilde{\lambda}_{+}$. The quadratic equation then reduces to a linear one, from which

$\eta \approx \tilde{\lambda}_{+} \log \frac{-2\left(\Delta_{\perp} \hat{\phi}-\Delta \phi_{+}+\lambda_{-} \Delta \epsilon\right)}{\left(\frac{\Delta \phi_{+}}{\tilde{\lambda}_{+}}+\frac{\Delta \phi_{*}}{\tilde{\lambda}_{*}}\right)\left(\tilde{\lambda}_{+}+\lambda_{-}\right)}$.

The argument of the logarithm is positive for $\Delta_{\perp} \hat{\phi}<0$ and when $\Delta \epsilon$ is small enough. As $\Delta \phi_{*}$ and $\Delta \phi_{+}$become smaller for $j_{\mathrm{PO}} \rightarrow 0$, the transition point $\eta$ becomes progressively larger.

Figure 5, right column, shows the lobe-plasma sheet boundary for a precipitation/outflow offset current given by $j_{0}=+3 \mu \mathrm{A} / \mathrm{m}^{2}$ and $K_{0}=0.5 \mu \mathrm{A} / \mathrm{kV} \mathrm{m}^{2}$. The sign changes of $\Delta_{\|} \phi$ at $\xi=-0.3 \mathrm{~km}$ and $\eta=26.0 \mathrm{~km}$ determine the limited extent of the region where the ionospheric ions can escape. The numerical solution of the nonlinear equations for $\xi$ and $\eta$ has been simplified by using the values found for $\xi$ in the absence of precipitation/outflow offset current (Sect. 3.1.1) and for $\eta$ in the thin interface limit (derived above) as initial guesses. The ionospheric potential (panel a, blue) is below the magnetospheric potential (panel a, green) as $x \rightarrow+\infty$. The field-aligned potential difference $\Delta_{\|} \phi$ is negative on the lobe side, changes sign at $\xi$, becomes positive on the plasma sheet side, until it turns negative again at $\eta$, after which it asymptotically reaches the value $\Delta \phi_{*}$ (panel b). Since the parallel potential difference now is a bit larger when compared to the case $j_{\mathrm{PO}}=0$, the field-aligned currents are somewhat stronger and the ionospheric perpendicular electric field and the Pedersen current are more intense (panels $\mathrm{c}-\mathrm{g}$ ). The outflowing ionospheric ions now have to overcome an electric potential barrier for $x>\eta$. If their initial energy $W_{0}$ exceeds $Z_{+} e \Delta_{\|} \phi$, they can still escape but their parallel velocity is reduced; if $W_{0}$ is insufficient to overcome the barrier, then the outflow region has only a limited extent, as in the present example (panels $\mathrm{h}-\mathrm{j}$ ).

\subsection{Monopolar field with $\Delta_{\perp} \hat{\phi} \geq 0$}

The auroral current circuit has a completely different structure for the opposite sign of the potential difference across the interface, $\Delta_{\perp} \hat{\phi} \geq 0$. We will address this situation only with the basic field-aligned current model.

\subsubsection{Basic field-aligned current model, $\Delta \epsilon>0$}

If $\Delta \epsilon>0$, the parallel potential difference must be positive for $x<0$ and negative for $x>0$, with a sign change at a point $\xi$ inside the boundary. Following the basic field-aligned current model, the parallel currents above the auroral oval and the equatorward part of the boundary, where $\Delta_{\|} \phi<0$, are

$j_{\|}=\left(K_{\mathrm{i}-}-K_{\mathrm{i}+}+K_{\mathrm{m}+}\right) \Delta_{\|} \phi=K_{*} \Delta_{\|} \phi$.

With $\Delta_{\|} \phi>0$, the parallel currents are

$j_{\|}=K_{\mathrm{m}-} \Delta_{\|} \phi=K_{+} \Delta_{\|} \phi$

above the poleward part of the boundary. The lobe and the polar cap are not able to supply significant current for $\Delta_{\|} \phi>0$, resulting in a small Knight constant as there are not enough lobe particles to maintain a strong precipitation (the lobe density may be $0.1-0.5$ particles per $\mathrm{cm}^{3}$ (Engwall et al., 2006), but their low energies correspond to a narrow loss cone and to speeds that are too slow to provide a significant flux) nor ionospheric particles to flow upward (negligible precipitation implies that the upper ionosphere is not heated; also, the sign of the parallel potential is such that it now has to accelerate ions rather than electrons out of the ionosphere, which is much harder because of their mass). A small Knight constant is equivalent to a long length scale. As long as this length scale exceeds the size of the region considered here, the Knight constant is effectively zero and

$j_{\|}=0$ 

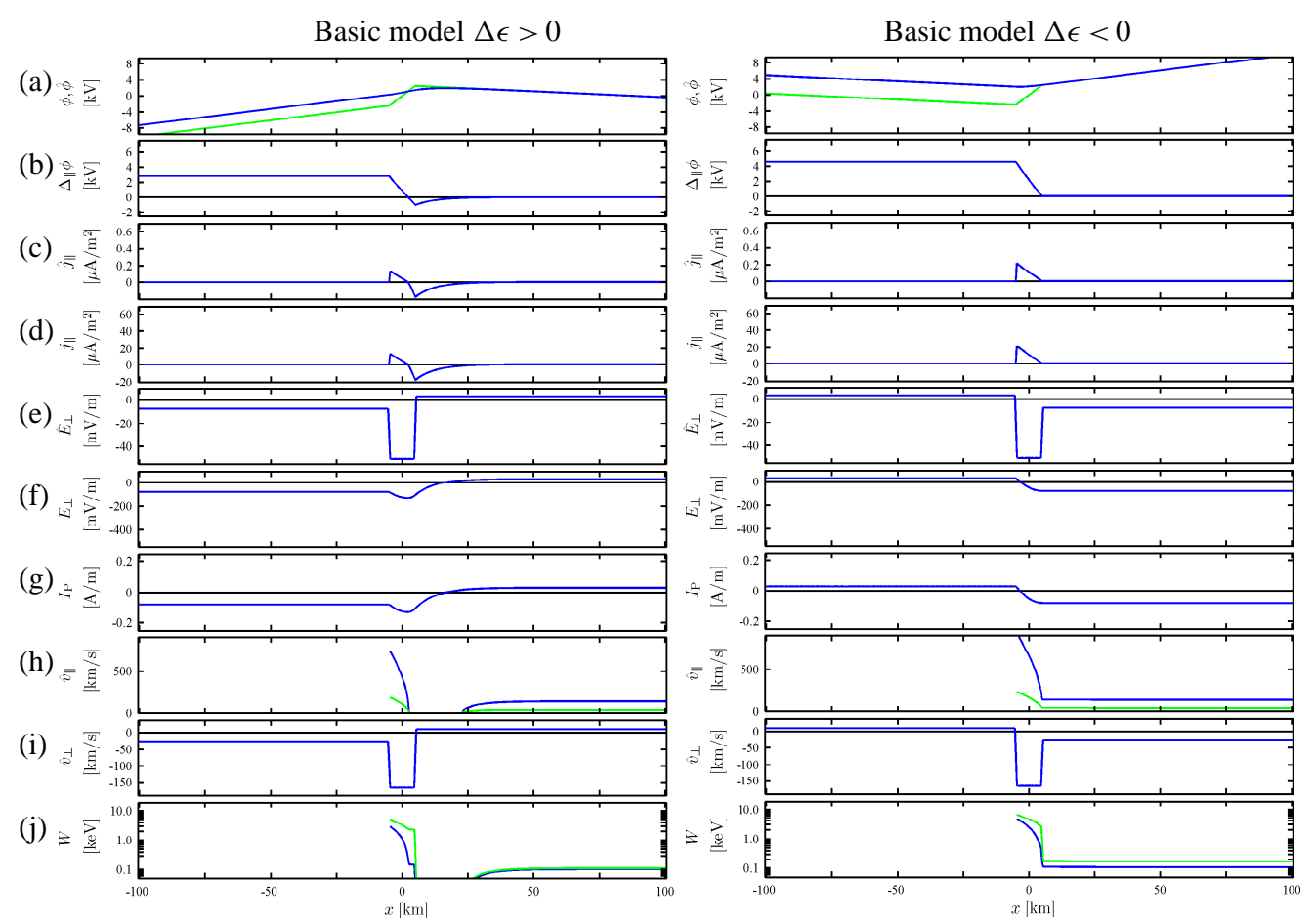

Fig. 6. Auroral current structure for monopolar electric fields at a lobe-plasma sheet boundary with $\Delta_{\perp} \hat{\phi}=+5 \mathrm{kV}$. The boundary thickness is $10 \mathrm{~km}$ at ionospheric altitude. The basic field-aligned current model was used. Left: $\epsilon_{\mathrm{L}}=-80 \mathrm{mV} / \mathrm{m}$ and $\epsilon_{\mathrm{R}}=30 \mathrm{mV} / \mathrm{m}$. Right: $\epsilon_{\mathrm{L}}=30 \mathrm{mV} / \mathrm{m}$ and $\epsilon_{\mathrm{R}}=-80 \mathrm{mV} / \mathrm{m}$.

above the polar cap. Current continuity can now be expressed as

$$
\frac{\mathrm{d}^{2} \phi}{\mathrm{d} x^{2}}=\left\{\begin{array}{lr}
0, & x<-P, \\
\frac{1}{\lambda_{+}^{2}}(\phi-\hat{\phi}), & -P \leq x<\xi, \\
\frac{1}{\lambda_{*}^{2}}(\phi-\hat{\phi}), & \xi \leq x .
\end{array}\right.
$$

Solving this ordinary differential equation piecewise gives the ionospheric potential in terms of $\xi$, which is found from $\Delta_{\|} \phi(\xi)=0$.

It is again illustrative to consider the thin interface limit, in which the ionospheric potential is

$\phi=\hat{\phi}+ \begin{cases}\Delta_{\perp} \hat{\phi}-\lambda_{*} \Delta \epsilon, & x<0, \\ -\lambda_{*} \Delta \epsilon \mathrm{e}^{-x / \lambda_{*},} & 0 \leq x .\end{cases}$

The ionospheric potential above the polar cap varies linearly with $x$, with the same slope as $\hat{\phi}$. Obviously, $\lambda_{+}$does not intervene in the thin interface solution.

The left panel of Fig. 6 shows a typical solution for $\Delta_{\perp} \hat{\phi}=5 \mathrm{kV}$ and for a $P=5 \mathrm{~km}$ thick interface. The parallel potential difference (panel b) is large above the polar cap, on the order of $\Delta_{\perp} \hat{\phi}$. In the example the convection electric fields were chosen rather large to obtain a clear figure, $\epsilon_{\mathrm{L}}=-80 \mathrm{mV} / \mathrm{m}$ and $\epsilon_{\mathrm{R}}=30 \mathrm{mV} / \mathrm{m}$. A strong field-aligned current appears inside the boundary, near its poleward border (panels $\mathrm{c}$ and $\mathrm{d}$, between $-P=-5 \mathrm{~km}$ and $\xi=2.2 \mathrm{~km}$ ). This current layer is associated with a narrow region of magnetospheric electron precipitation and upward accelerated ionospheric ions. It is separated by a gap from the continuous precipitation and upflow above the auroral oval. The precipitating electrons come from the poleward edge of the plasma sheet population, which pervades the interface. In the simple description used here, the density of the plasma sheet particles drops abruptly at that poleward edge, while in reality a smooth change in density is expected across the interface over a thickness scale dictated by the particle gyroradius. Above the auroral oval, away from its poleward border, there is ionospheric ion outflow (panel $h$ ) but these ions are not accelerated beyond their initial energy $W_{0}$ (panel j).

\subsubsection{Basic field-aligned current model, $\Delta \epsilon<0$}

If $\Delta \epsilon<0$, it turns out that several configurations are possible, depending on the values of $\epsilon_{\mathrm{L}}, \epsilon_{\mathrm{R}}, P$, and $\Delta_{\perp} \hat{\phi}$. Only one of them will be analyzed here. Consider the situation where $\Delta_{\|} \phi>0$ everywhere. The field-aligned currents above the auroral oval and its boundary are

$j_{\|}=K_{\mathrm{m}-} \Delta_{\|} \phi=K_{+} \Delta_{\|} \phi$,

while above the polar cap

$j_{\|}=0$. 


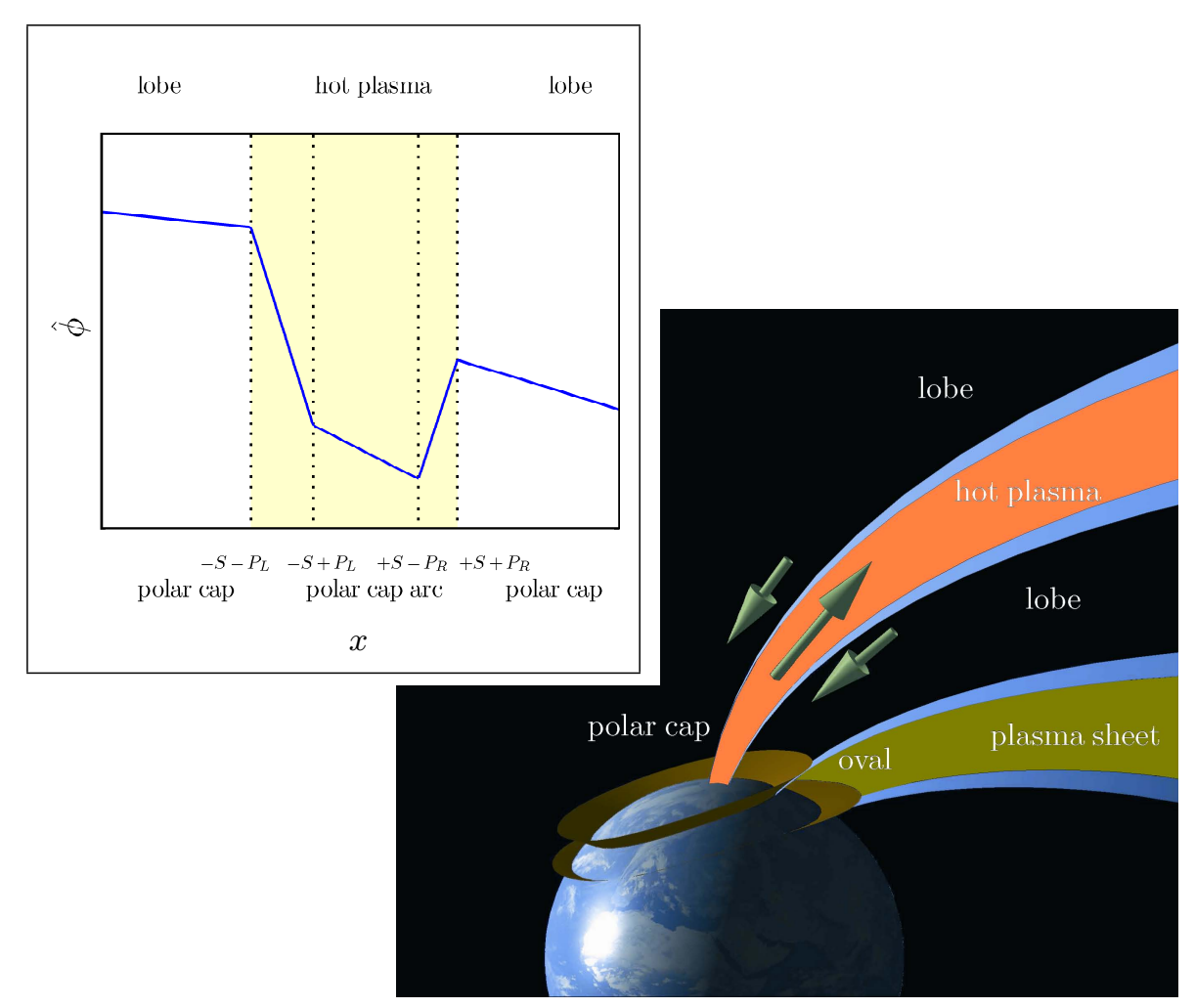

Fig. 7. Various configurations can give rise to bipolar auroral electric fields. This sketch shows the geometry for a slab of plasma sheetlike hot plasma (orange), embedded in the cold lobe medium (black) and separated from the plasma sheet itself (green). Such an entity would map into the polar cap, and could give rise to a polar cap arc. Up- and downward currents (represented by arrows) couple the magnetospheric structure to its ionospheric signature. The inset shows the one-dimensional situation considered here. The blue curve gives the magnetospheric electric potential $\hat{\phi}$ as a function of position across the structure, from pole to equator. The shaded region indicates where hot ion and electron precipitation and ionospheric ion outflow occur. Hot ion and electron precipitation extend throughout both interfaces.

The solution can again be computed from the current continuity condition. The ionospheric potential in the thin interface limit is

$$
\phi=\hat{\phi}+ \begin{cases}\Delta_{\perp} \hat{\phi}-\lambda_{+} \Delta \epsilon, & x<0, \\ -\lambda_{+} \Delta \epsilon \mathrm{e}^{-x / \lambda_{+},} & 0 \leq x,\end{cases}
$$

which is very similar to the previous case. As shown in the right panel of Fig. 6, a field-aligned current appears at the poleward edge of the interface and the gap between the outflow in the polar cap boundary and above the oval has disappeared.

\section{Bipolar electric fields}

Turning to the case of bipolar electric fields, the number of possible configurations increases dramatically. The discussion is limited here to situations where hot plasma sheet plasma is embedded in colder plasma sheet or lobe material (Fig. 7). The medium on either side of the structure is assumed to be identical, and the two potential jumps $\Delta_{\perp} \hat{\phi}_{\mathrm{L}}$ and $\Delta_{\perp} \hat{\phi}_{\mathrm{R}}$ on either side have the same order of magnitude.

\subsection{Bipolar field with $\Delta_{\perp} \hat{\phi}_{\mathrm{L}}<0$ and $\Delta_{\perp} \hat{\phi}_{\mathrm{R}}>0$}

Consider a pair of interfaces, separated by a distance $2 S$. The left interface has half-thickness $P_{\mathrm{L}}$, the one at the right $P_{\mathrm{R}}$. This situation can be represented by a magnetospheric potential profile of the form (Fig. 7)

$$
\hat{\phi}=\left\{\begin{array}{c}
-\Delta_{\perp} \hat{\phi}_{\mathrm{L}}+\epsilon_{\mathrm{C}}\left(S-P_{\mathrm{L}}\right)-\epsilon_{\mathrm{L}}\left(x+S+P_{\mathrm{L}}\right), \\
x<-S-P_{\mathrm{L}}, \\
+\epsilon_{\mathrm{C}}\left(S-P_{\mathrm{L}}\right)+\frac{\Delta_{\perp} \hat{\phi}_{\mathrm{L}}}{2 P_{\mathrm{L}}}\left(x+S-P_{\mathrm{L}}\right), \\
-S-P_{\mathrm{L}} \leq x<-S+P_{\mathrm{L}}, \\
-S+P_{\mathrm{L}} \leq x<+S-P_{\mathrm{R}}, \\
-\epsilon_{\mathrm{C}}\left(S-P_{\mathrm{R}}\right)+\frac{\Delta_{\perp} \hat{\phi}_{\mathrm{R}}}{2 P_{\mathrm{R}}}\left(x-S+P_{\mathrm{R}}\right), \\
+S-P_{\mathrm{R}} \leq x<+S+P_{\mathrm{R}}, \\
+\Delta_{\perp} \hat{\phi}_{\mathrm{R}}-\epsilon_{\mathrm{C}}\left(S-P_{\mathrm{R}}\right)-\epsilon_{\mathrm{R}}\left(x-S-P_{\mathrm{R}}\right), \\
+S+P_{\mathrm{R}} \leq x,
\end{array}\right.
$$

where $\hat{\phi}(0)=0$. The size of the structure, $S$, is larger than the interface thicknesses but smaller than or on the order of the characteristic auroral length scales $\left(\lambda_{-}, \lambda_{*}, \lambda_{+}\right)$; if not, it 
would be more appropriate to view the two potential jumps as individual monopolar transitions. Small convection electric fields $\epsilon_{\mathrm{L}}, \epsilon_{\mathrm{C}}$, and $\epsilon_{\mathrm{R}}$ may be present. When $\Delta_{\perp} \hat{\phi}_{\mathrm{L}}<0(>0)$ and $\Delta_{\perp} \hat{\phi}_{R}>0(<0)$, the parallel potential difference is negative (positive) outside the structure and positive (negative) within.

\subsubsection{Basic field-aligned current model}

The parallel potential changes from negative to positive at some point $\xi$ in the left interface, and from positive to negative at $\eta$ in the right interface, so that

$$
\frac{\mathrm{d}^{2} \phi}{\mathrm{d} x^{2}}= \begin{cases}\frac{1}{\lambda_{-}^{2}}(\phi-\hat{\phi}), & x<-S-P_{\mathrm{L}}, \\ \frac{1}{\lambda_{*}^{2}}(\phi-\hat{\phi}), & -S-P_{\mathrm{L}} \leq x<\xi \\ \frac{1}{\lambda_{+}^{2}}(\phi-\hat{\phi}), & \xi \leq x<\eta \\ \frac{1}{\lambda_{*}^{2}}(\phi-\hat{\phi}), & \eta \leq x<+S+P_{\mathrm{R}} \\ \frac{1}{\lambda_{-}^{2}}(\phi-\hat{\phi}), & +S+P_{\mathrm{R}} \leq x\end{cases}
$$

The same solution strategy applies as before, with $\Delta_{\|} \phi(\xi)=$ 0 and $\Delta_{\|} \phi(\eta)=0$ forming a set of two nonlinear algebraic equations from which $\xi$ and $\eta$ are obtained. The analytical form of the solution is not given here as it is too complicated to be useful.

It is again instructive to look at the thin interface limit, when $\xi=-S$ and $\eta=+S$. Using the boundary conditions and requiring continuity of $\phi(x)$ and $I_{\mathrm{P}}(x)$ at $-S$ and $+S$, and solving the system, results in

$$
\phi=\hat{\phi}+\left\{\begin{array}{lc}
\left(\alpha+\Delta_{\perp} \hat{\phi}_{\mathrm{L}}\right) \mathrm{e}^{(x+S) / \lambda_{-}}, & x<-P, \\
\frac{\beta \mathrm{e}^{2 S / \lambda_{+}-\alpha}}{\mathrm{e}^{4 S / \lambda_{+}-1}} \mathrm{e}^{(x+S) / \lambda_{+}}+\frac{\alpha \mathrm{e}^{2 S / \lambda_{+}-\beta}}{\mathrm{e}^{4 S / \lambda_{+}-1}} \mathrm{e}^{-(x-S) / \lambda_{+},} & -P \leq x<+P, \\
\left(\beta-\Delta_{\perp} \hat{\phi}_{\mathrm{R}}\right) \mathrm{e}^{-(x-S) / \lambda_{-}}, & +P \leq x,
\end{array}\right.
$$

where the field-aligned potential differences just inward of the left and right boundaries are

$$
\begin{aligned}
\alpha= & \lambda_{-}\left(\Delta_{\perp} \hat{\phi}_{\mathrm{L}}\left[\mathrm{e}^{4 S / \lambda_{+}}\left(\lambda_{+}+\lambda_{-}\right)+\left(\lambda_{+}-\lambda_{-}\right)\right]\right. \\
& -\lambda_{+} \Delta \epsilon_{\mathrm{L}}\left[\mathrm{e}^{4 S / \lambda_{+}}\left(\lambda_{+}+\lambda_{-}\right)-\left(\lambda_{+}-\lambda_{-}\right)\right] \\
& \left.+2 \mathrm{e}^{2 S / \lambda_{+}} \lambda_{+}\left(\Delta_{\perp} \hat{\phi}_{\mathrm{R}}+\lambda_{-} \Delta \epsilon_{\mathrm{R}}\right)\right) / \kappa-\Delta_{\perp} \hat{\phi}_{\mathrm{L}}, \\
\beta= & \lambda_{-}\left(\Delta_{\perp} \hat{\phi}_{\mathrm{R}}\left[\mathrm{e}^{4 S / \lambda_{+}}\left(\lambda_{+}+\lambda_{-}\right)+\left(\lambda_{+}-\lambda_{-}\right)\right]\right. \\
& +\lambda_{+} \Delta \epsilon_{\mathrm{R}}\left[\mathrm{e}^{4 S / \lambda_{+}}\left(\lambda_{+}+\lambda_{-}\right)-\left(\lambda_{+}-\lambda_{-}\right)\right] \\
& \left.-2 \mathrm{e}^{2 S / \lambda_{+}} \lambda_{+}\left(\Delta_{\perp} \hat{\phi}_{\mathrm{L}}+\lambda_{-} \Delta \epsilon_{\mathrm{L}}\right)\right) / \kappa+\Delta_{\perp} \hat{\phi}_{\mathrm{R}},
\end{aligned}
$$

where $\kappa=\mathrm{e}^{4 S / \lambda_{+}}\left(\lambda_{+}+\lambda_{-}\right)^{2}-\left(\lambda_{+}-\lambda_{-}\right)^{2}$ and where $\Delta \epsilon_{\mathrm{L}}=$ $\epsilon_{\mathrm{C}}-\epsilon_{\mathrm{L}}$ and $\Delta \epsilon_{\mathrm{R}}=\epsilon_{\mathrm{C}}-\epsilon_{\mathrm{R}}$. For $S \gg \lambda_{+}$, the terms in $\mathrm{e}^{4 S / \lambda_{+}}$ dominate. The terms involving $\Delta_{\perp} \hat{\phi}_{\mathrm{R}}$ and $\Delta \epsilon_{\mathrm{R}}$ in the expression for $\alpha$, and those involving $\Delta_{\perp} \hat{\phi}_{\mathrm{L}}$ and $\Delta \epsilon_{\mathrm{L}}$ in the expression for $\beta$, then become irrelevant: The parallel potential at one boundary does not depend on the potential jump at the other boundary. As expected, the two problems decouple completely for $S \rightarrow \infty$, as Eq. (24) then reduces to two separate instantiations of Eq. (10).

When $\lambda_{-} \ll S$, that is, when $K_{-}$is very large, a simpler form of solution is found, with $\alpha=-\Delta_{\perp} \hat{\phi}_{\mathrm{L}}$ and $\beta=\Delta_{\perp} \hat{\phi}_{\mathrm{R}}$, so that

$$
\begin{aligned}
\phi=\hat{\phi} & +\frac{\Delta_{\perp} \hat{\phi}_{\mathrm{R}} \mathrm{e}^{2 S / \lambda_{+}}+\Delta_{\perp} \hat{\phi}_{\mathrm{L}}}{\mathrm{e}^{4 S / \lambda_{+}}-1} \mathrm{e}^{(x+S) / \lambda_{+}} \\
& -\frac{\Delta_{\perp} \hat{\phi}_{\mathrm{L}} \mathrm{e}^{2 S / \lambda_{+}}+\Delta_{\perp} \hat{\phi}_{\mathrm{R}}}{\mathrm{e}^{4 S / \lambda_{+}}-1} \mathrm{e}^{-(x-S) / \lambda_{+}}
\end{aligned}
$$

inside the central region and $\phi=\hat{\phi}$ outside. The upward current clearly extends throughout the embedded region, with impulse-like return current regions on either side. The auroral current circuit then is completely characterized by the $\hat{\phi}$-profile and by $\lambda_{+}$(or, equivalently, $K_{+}$). Using the symmetric version of this simple solution, with $\Delta_{\perp} \hat{\phi}=-\Delta_{\perp} \hat{\phi}_{\mathrm{L}}=$ $+\Delta_{\perp} \hat{\phi}_{R}$, it is easy to assess the thickness above which the bipolar structure can be considered as two adjacent monopolar ones. The relative magnitude of the parallel potential difference at the center is

$$
\frac{\Delta_{\|} \phi(0)}{\Delta_{\perp} \hat{\phi}}=\frac{\phi(0)-\hat{\phi}(0)}{\Delta_{\perp} \hat{\phi}}=\frac{1}{\cosh \left(2 S / \lambda_{+}\right)},
$$

so that $\Delta_{\|} \phi(0)$ is less than a fraction $\psi$ of the maximum potential difference $\Delta_{\perp} \hat{\phi}$ for a thickness $2 S>\lambda_{+} \operatorname{arccosh}(1 / \psi)$. For instance, for $\psi=0.1$, the thickness must exceed $2.993 \lambda_{+}$.

Figure 8, left panel, shows the solution for a bipolar configuration with $\Delta_{\perp} \hat{\phi}_{\mathrm{L}}=-5 \mathrm{kV}$ and $\Delta_{\perp} \hat{\phi}_{\mathrm{R}}=+3 \mathrm{kV}$, $\epsilon_{\mathrm{L}}=10 \mathrm{mV} / \mathrm{m}, \epsilon_{\mathrm{C}}=50 \mathrm{mV} / \mathrm{m}$, and $\epsilon_{\mathrm{R}}=-30 \mathrm{mV} / \mathrm{m}$, and with $K_{-}=15 \mu \mathrm{A} / \mathrm{kV} \mathrm{m}^{2}, K_{*}=17 \mu \mathrm{A} / \mathrm{kV} \mathrm{m}^{2}$, and $K_{+}=$ $5 \mu \mathrm{A} / \mathrm{kV} \mathrm{m}^{2}$ as before. The interface half-thicknesses are $P_{\mathrm{L}}=4 \mathrm{~km}$ and $P_{\mathrm{R}}=5 \mathrm{~km}$, less than the embedded region thickness, but of the same order. A positive $\Delta_{\|} \phi$ is found in the hot plasma region, which is $2 S=40 \mathrm{~km}$ wide. The parallel potential changes sign at $\xi=-21.0 \mathrm{~km}$ and $\eta=23.1 \mathrm{~km}$. Hot electron precipitation is fairly intense in the inner region, with accelerating potentials $\alpha$ and $\beta$ of the order of $\Delta_{\perp} \hat{\phi}_{\mathrm{L}}$ and $\Delta_{\perp} \hat{\phi}_{\mathrm{R}}$ (panels a and $\mathrm{b}$ ). Since the energy of the hot ions exceeds $\Delta_{\perp} \hat{\phi}_{\mathrm{L}}$ and $\Delta_{\perp} \hat{\phi}_{\mathrm{R}}$ (if not, such tangential discontinuities could not exist), they are able to precipitate as well despite the fact that $\Delta_{\|} \phi$ decelerates them. Given the fact that the hot particle precipitation extends throughout the interfaces, upflowing ionospheric ions are found over a region that is slightly broader than $[\xi, \eta]$, as just outside this region the ions have an energy $W_{0}$ that enables them to overcome even a slight negative $\Delta_{\|} \phi$. Upward field-aligned currents flow in the embedded plasma region, while return currents flow on either side (panels $c$ and d). The bipolar perpendicular electric field at magnetospheric altitude is characterized by two pulses of opposite sign (panel e), while the ionospheric $E_{\perp}$ alternates sign more smoothly across the bipolar 

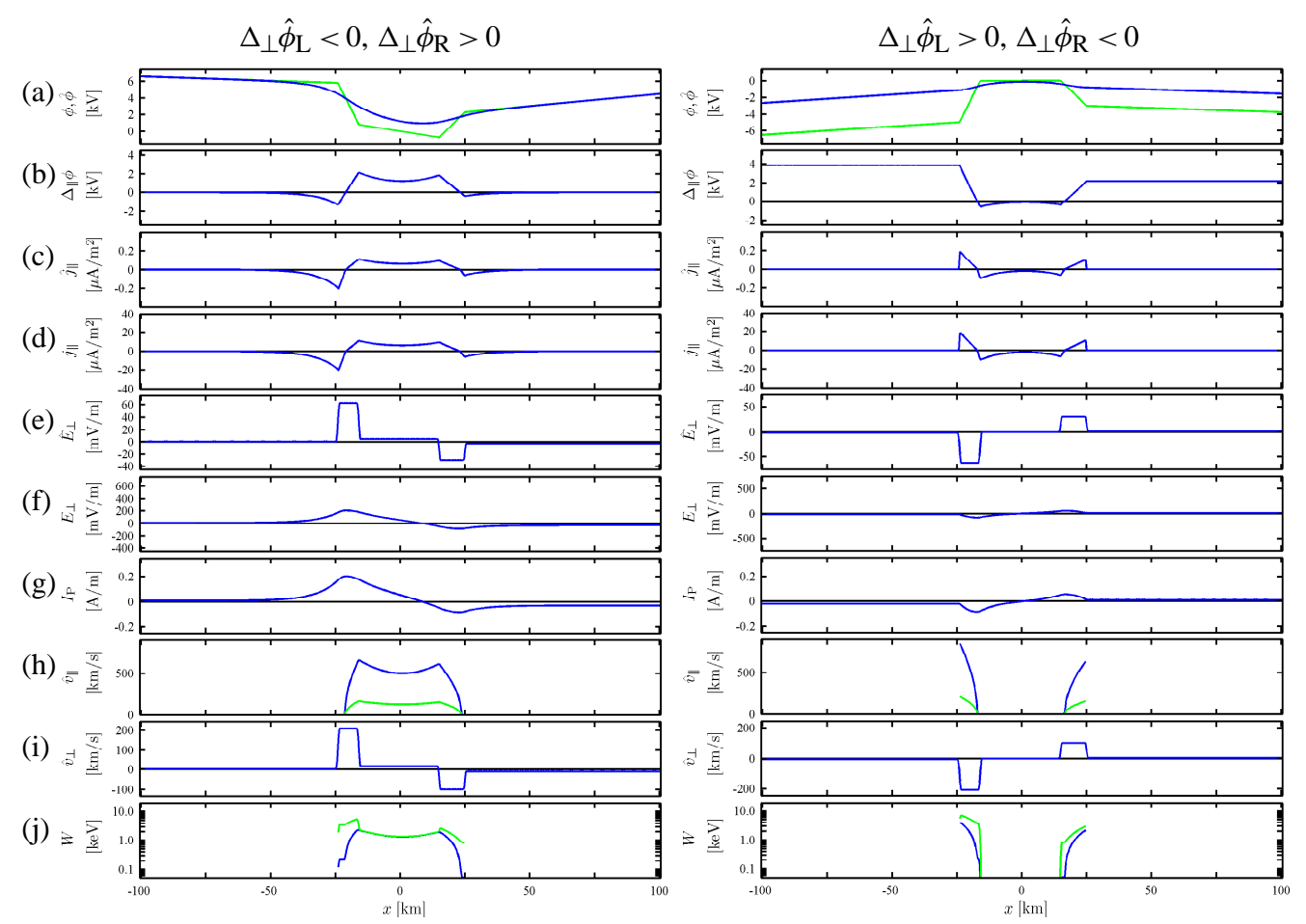

Fig. 8. Auroral current structure for bipolar electric fields that correspond to a $40 \mathrm{~km}$ wide region ( $400 \mathrm{~km}$ in the magnetosphere) of plasma sheet material embedded in the lobe. The left and right interfaces have thicknesses of 8 and $10 \mathrm{~km}$. Left: for electric potential jumps $\Delta_{\perp} \hat{\phi}_{\mathrm{L}}=-5 \mathrm{kV}$ and $\Delta_{\perp} \hat{\phi}_{\mathrm{R}}=+3 \mathrm{kV}$ and convection electric fields $\epsilon_{\mathrm{L}}=10 \mathrm{mV} / \mathrm{m}, \epsilon_{\mathrm{C}}=50 \mathrm{mV} / \mathrm{m}$, and $\epsilon_{\mathrm{R}}=-30 \mathrm{mV} / \mathrm{m}$. Right: for electric potential jumps $\Delta_{\perp} \hat{\phi}_{\mathrm{L}}=+5 \mathrm{kV}$ and $\Delta_{\perp} \hat{\phi}_{\mathrm{R}}=-3 \mathrm{kV}$. The convection electric fields are $\epsilon_{\mathrm{L}}=-20 \mathrm{mV} / \mathrm{m}, \epsilon_{\mathrm{C}}=0 \mathrm{mV} / \mathrm{m}$, and $\epsilon_{\mathrm{R}}=10 \mathrm{mV} / \mathrm{m}$.

electric field structure (panel f). The profile of the Pedersen current illustrates that the upward and downward currents across each of both interfaces more or less balance each other (panel g). Ionospheric ions are accelerated upward and acquire a substantial parallel velocity (panel $h$ ). The particles acquire a perpendicular $\boldsymbol{E} \times \boldsymbol{B}$ drift velocity (pitch angles $90^{\circ}$ ) of opposite sign at the two interfaces (panel i). Inside the embedded plasma sheet region, the upgoing ion energy is mainly parallel (pitch angle $0^{\circ}$ or $180^{\circ}$ depending on the hemisphere), as the ions there gain a significant parallel energy by field-aligned acceleration, on top of their initial energy $W_{0}$ (panel j).

\subsubsection{Advanced field-aligned current model}

It is possible to address the same problem with the advanced field-aligned current model. For a negative $j_{\mathrm{PO}}$, the sign of $\Delta_{\|} \phi$ is positive in the embedded region, so that the same type of solution remains valid. For a positive $j_{\mathrm{PO}}$, the parallel potential $\Delta_{\|} \phi$ can in principle become negative in the embedded region. However, if $S$ is of the order of $\lambda_{+}$and if $j_{0}$ is small, this never happens as the length scale associated with $j_{0}$ is much wider than the structure. A small $j_{\mathrm{PO}}$ therefore does not significantly affect the solution.

\subsection{Bipolar field with $\Delta_{\perp} \hat{\phi}_{\mathrm{L}}>0$ and $\Delta_{\perp} \hat{\phi}_{\mathrm{R}}<0$}

If the magnetospheric potential jumps have the opposite sign as before, $\Delta_{\perp} \hat{\phi}_{\mathrm{L}}>0$ and $\Delta_{\perp} \hat{\phi}_{\mathrm{R}}<0$, different configurations are possible depending on the signs of $\Delta \epsilon_{\mathrm{L}}$ and $\Delta \epsilon_{\mathrm{R}}$. As an example, the discussion here addresses the case $\Delta \epsilon_{\mathrm{L}}>0$ and $\Delta \epsilon_{\mathrm{R}}<0$, with small convection electric fields, so that the parallel potential difference is negative in the embedded layer.

\subsubsection{Basic field-aligned current model}

Given that $\Delta_{\|} \phi<0$ between points $\xi$ and $\eta$ located inside the interfaces, and $\Delta_{\|} \phi>0$ outside, current continuity based on the basic field-aligned current model dictates that

$$
\frac{\mathrm{d}^{2} \phi}{\mathrm{d} x^{2}}= \begin{cases}0, & x<-S-P_{\mathrm{L}}, \\ \frac{1}{\lambda_{+}^{2}}(\phi-\hat{\phi}), & -S-P_{\mathrm{L}} \leq x<\xi, \\ \frac{1}{\lambda_{*}^{2}}(\phi-\hat{\phi}), & \xi \leq x<\eta, \\ \frac{1}{\lambda_{+}^{2}}(\phi-\hat{\phi}), & \eta \leq x<+S+P_{\mathrm{R}}, \\ 0, & +S+P_{\mathrm{R}} \leq x .\end{cases}
$$

For the sake of simplicity, we turn again to the thin interface limit where $\xi=-S$ and $\eta=+S$. The ionospheric potential 
then is

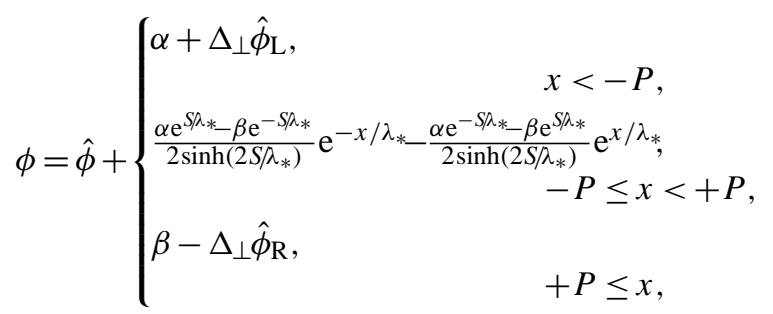

where the field-aligned potential differences just inward of the left and right boundaries are

$$
\begin{aligned}
& \alpha=-\frac{\lambda_{*}}{\tanh \left(2 S / \lambda_{*}\right)}\left(\Delta \epsilon_{\mathrm{L}}-\frac{\Delta \epsilon_{\mathrm{R}}}{\cosh \left(2 S / \lambda_{*}\right)}\right), \\
& \beta=+\frac{\lambda_{*}}{\tanh \left(2 S / \lambda_{*}\right)}\left(\Delta \epsilon_{\mathrm{R}}-\frac{\Delta \epsilon_{\mathrm{L}}}{\cosh \left(2 S / \lambda_{*}\right)}\right) .
\end{aligned}
$$

The above expressions for $\alpha$ and $\beta$ illustrate how $\alpha$ becomes independent of $\Delta \epsilon_{\mathrm{R}}$ and $\beta$ of $\Delta \epsilon_{\mathrm{L}}$ for large $S / \lambda_{*}$, as the electric field structure then consists of two individual monopolar transitions.

Figure 8, right column, shows the solution for thick boundaries $\left(P_{\mathrm{L}}=4 \mathrm{~km}\right.$ and $\left.P_{\mathrm{R}}=5 \mathrm{~km}\right)$. The parallel potential difference changes sign at points $\xi=-17.1 \mathrm{~km}$ and $\eta=16.6 \mathrm{~km}$. Since the hot particle precipitation is present throughout the two interfaces, upward current regions with electron precipitation and upgoing ions are present at the outer edges of the interfaces; the return current is situated below the core of the embedded structure.

\subsubsection{Advanced field-aligned current model}

It is again possible to address this problem with the advanced field-aligned current model. Since, however, the field-aligned currents are still considered negligible outside the embedded structure, there will only be a small effect on the solution inside the structure. If the embedded region is narrow enough, that effect is negligible.

\section{Conclusions}

Auroral current circuits are associated with magnetospheric interfaces (Roth et al., 1993; Marghitu et al., 2006; Echim et al., 2007, 2009; Johansson et al., 2006). The hypothesis made here is that an external potential difference may exist across such interfaces (as in Roth et al., 1993). A different, but essentially equivalent assumption is that there may be velocity shears across them (as in De Keyser, 1999; Echim et al., 2007). Based on fundamental properties of field-aligned plasma interfaces (tangential discontinuities), this paper has demonstrated that these potential differences give rise to strong perpendicular electric fields inside the interfaces, of monopolar and bipolar type, or even more complicated. In addition, an analysis of auroral current circuit structure associated with these monopolar and bipolar electric fields has been presented. The analysis is based on the principle of current continuity in the context of an electrostatic model, so that the horizontal ionospheric current can be related to the field-aligned currents in- and out of the ionosphere. This allows the computation of the field-aligned potential differences, the parallel currents, and the properties of escaping ionospheric ions. The simplifications (ad hoc profile of the magnetospheric electric potential, constant conductivity, piecewise linear form of the current-voltage relation, ...) are such that the essential properties of the system are retained (see also De Keyser and Echim, 2010).

Three possibilities have been considered:

1. Hot plasma sheet material in contact with cold lobe plasma: This is exactly the situation at the plasma sheet boundary layer (the polar cap boundary). It has been shown that it is quite natural that a significant external potential difference appears across this layer. Kinetic model simulations illustrate that this gives rise to monopolar electric fields. The association of monopolar fields with the polar cap boundary has been established observationally (Johansson et al., 2006). An analysis of the electric structure shows that, for an equatorward monopolar field, the hot particles can always precipitate so that they can heat up the ionosphere and lead to the escape of ionospheric ions. For a poleward electric field, precipitation and ion escape are typically weaker and sometimes even precluded.

2. Hot plasma sheet material embedded in cold lobe plasma: this produces a pair of monopolar fields that may be so close together that one should treat them as a bipolar field structure. This can be a model for (some types of) polar cap arcs (Maggiolo et al., 2006). For electric field pairs with an equatorward field on the poleward edge and poleward field on the equatorward edge, hot magnetospheric ions are able to precipitate throughout the embedded region, which is exactly what is observed in polar cap arcs. For the opposite sign of the electric fields, electron precipitation and ion outflow is much less pronounced or even absent.

3. Different hot plasmas in contact with each other: This occurs at interfaces inside the plasma sheet. Such internal structure may arise during times of activity in the tail. In this case, bipolar or even more complicated electric field structures appear. The regions of opposite electric field then are always close to each other, at a distance of a few hot particle gyroradii at most. The predominant association of bipolar fields with the interior plasma sheet has also been found observationally (Johansson et al., 2006).

Note that there may be magnetospheric sources of monopolar or bipolar electric fields other than those in the plasma sheet 
or at the plasma sheet boundary, for instance, in the LLBL (Echim et al., 2008). The same principles govern the electric structure of such interfaces to the extent that they can be modelled as tangential discontinuities.

Monopolar and bipolar electric fields give rise to compound ionospheric structures, that include both upward and downward current regions. This implies that one finds characteristics of both discrete arcs (in the upward current regions) and of black aurorae (in the return current regions). For instance, at the polar cap boundary an intense arc is expected at the poleward edge of the auroral oval, adjacent to a black aurora at the equatorward edge of the polar cap. In narrow bipolar field structures, several successive up- and downward current regions may be present.

Hot plasma sheet particle precipitation often occurs throughout the auroral oval, with stronger emission typically at its poleward border due to large potential differences at the plasma sheet boundary. During substorms, internal structure may develop in the plasma sheet, giving rise to the subsequent appearance of discrete arcs inside the oval. Our analysis of the various configurations indicates that this precipitation very often leads to a significant outflow of ionospheric ions. This must be a major source for the $\mathrm{H}^{+}$and $\mathrm{O}^{+}$circulation in the magnetosphere (Chappell et al., 1987; Moore et al., 1999). Another general conclusion is that, for many of these auroral structures, the ionospheric electric field is strong enough to produce ionospheric drift and convection along the auroral arc, thus contributing to ionospheric plasma convection in the auroral zone (Kullen et al., 2008).

The analysis presented here relies on an electrostatic model. The magnetospheric configuration varies in time, although the structure often persists over a time scale of minutes (e.g. Figueiredo et al., 2005). That is sufficient to establish the quasi-electrostatic auroral current circuits discussed here. Major time-dependent effects include, but are not limited to, (1) low-frequency waves communicating the field changes between the magnetosphere and the ionosphere (e.g. Rönnmark and Hamrin, 2000); (2) ionospheric conductivity variations due to precipitation, ionospheric drift, and neutral winds (Lyons, 1981; Walterscheid and Lyons, 1992; Echim et al., 2008); (3) lower hybrid waves, generated in the upper ionosphere by the precipitation and responsible for transverse ion heating (Eliasson et al., 1994; Moore et al., 1999); (4) waves and instabilities due to interactions between the various up- and downgoing particle beams on the same field lines; (5) depletion and replenishment of the particles at the magnetospheric interface as discussed by Roth et al. (1993) and De Keyser (1999); and (6) enhanced wave activity providing the pitch-angle scattering needed for re-populating the loss cone (e.g. Sigsbee et al., 1998; Swift, 2001).

\section{Appendix A}

\section{Solution for monopolar field $\left(\Delta_{\perp} \hat{\phi}<0\right)$}

This Appendix gives the full analytic solution for the ionospheric potential in the case of a monopolar electric field with $\Delta_{\perp} \hat{\phi}<0$ at a thick interface (half-thickness $P$ ), using the basic field-aligned current model. This is the problem described in Sect. 3.1.1.

The solution can be expressed in terms of $\xi$, the point where the field-aligned potential difference changes sign. The condition $\Delta_{\|} \phi(\xi)=0$ corresponds to

$\Delta_{\perp} \hat{\phi}+2 \epsilon_{\mathrm{R}} P=\gamma\left(\Delta_{\perp} \hat{\phi}+2 \epsilon_{\mathrm{L}} P\right)$,

where

$\gamma=\frac{\lambda_{-} \mathrm{e}^{(P-\xi) / \lambda_{+}}}{\lambda_{-} \cosh \frac{\xi+P}{\lambda_{*}}+\lambda_{*} \sinh \frac{\xi+P}{\lambda_{*}}}$.

Solving this nonlinear equation for $\xi$, the ionospheric potential is found as

$$
\phi=\hat{\phi}+\left\{\begin{array}{rr}
\frac{\Delta_{\perp} \hat{\phi}+2 \epsilon_{\mathrm{L}} P}{2 P v} \lambda_{-} C_{1} \mathrm{e}^{(x+P) / \lambda_{-}}, & x<-P, \\
\frac{\Delta_{\perp} \hat{\phi}+2 \epsilon_{\mathrm{L}} P}{2 P v} \lambda_{*}\left(C_{2} \mathrm{e}^{-(x-P) / \lambda_{*}+}+C_{3} \mathrm{e}^{(x+P) / \lambda_{*}}\right), & -P \leq x<\xi, \\
\frac{\Delta_{\perp} \hat{\phi}+2 \epsilon_{\mathrm{R}} P}{4 P \nu} \lambda_{+}\left(C_{4} \mathrm{e}^{-(x+P) / \lambda_{*}+} C_{5} \mathrm{e}^{\left.(x-P) / \lambda_{*}\right),}\right. & \xi \leq x<+P, \\
\frac{\Delta_{\perp} \hat{\phi}+2 \epsilon_{\mathrm{R}} P}{4 P v} \lambda_{+} C_{6} \mathrm{e}^{-(x-P) / \lambda_{+}}, & +P \leq x,
\end{array}\right.
$$

with

$$
\begin{aligned}
v= & \left(\lambda_{*}+\lambda_{+}\right)\left(\lambda_{*}+\lambda_{-}\right) \sigma_{*}^{2} \tau_{*}^{2}-\left(\lambda_{*}-\lambda_{+}\right)\left(\lambda_{*}-\lambda_{-}\right), \\
C_{1}= & {\left[\left(\lambda_{+}-\lambda_{*}\right)+\left(\lambda_{+}+\lambda_{*}\right) \sigma_{*}^{2} \tau_{*}^{2}-2 \lambda_{+} \sigma_{*} \tau_{*} \sigma_{+} \gamma / \tau_{+}\right] \lambda_{*}, } \\
C_{2}= & \left(\lambda_{+}+\lambda_{*}\right) \lambda_{-} \sigma_{*}^{2}-\left(\lambda_{-}-\lambda_{*}\right) \lambda_{+} \sigma_{*} \sigma_{+} \gamma / \tau_{*} \tau_{+}, \\
C_{3}= & \left(\lambda_{+}-\lambda_{*}\right) \lambda_{-}-\left(\lambda_{-}+\lambda_{*}\right) \lambda_{+} \sigma_{*} \tau_{*} \sigma_{+} \gamma / \tau_{+}, \\
C_{4}= & \left(\lambda_{+}-\lambda_{*}\right)\left(\lambda_{-}+\lambda_{*}\right) \sigma_{*}^{2} \tau_{*}^{2} \sigma_{+}^{2}-\left(\lambda_{+}+\lambda_{*}\right)\left(\lambda_{-}-\lambda_{*}\right) \sigma_{+}^{2} \\
& +4 \lambda_{+} \lambda_{-} \sigma_{*} \tau_{*} \sigma_{+} \tau_{+} / \gamma, \\
C_{5}= & -1, \\
C_{6}= & \left(\lambda_{+}-\lambda_{*}\right)\left(\lambda_{-}-\lambda_{*}\right)-\left(\lambda_{+}+\lambda_{*}\right)\left(\lambda_{+}+\lambda_{-}\right) \sigma_{*}^{2} \tau_{*}^{2} \\
& -\left(\lambda_{+}+\lambda_{*}\right)\left(\lambda_{-}-\lambda_{*}\right) \sigma_{+}^{2} / \tau_{+}^{2} \\
& +\left(\lambda_{+}-\lambda_{*}\right)\left(\lambda_{-}+\lambda_{*}\right) \sigma_{*}^{2} \tau_{*}^{2} \sigma_{+}^{2} / \tau_{+}^{2} \\
& +4 \lambda_{-} \lambda_{*} \sigma_{*} \tau_{*} \sigma_{+} / \gamma \tau_{+},
\end{aligned}
$$

where $\sigma_{*}=\mathrm{e}^{\xi / \lambda_{*}}, \sigma_{+}=\mathrm{e}^{\xi / \lambda_{+}}, \tau_{*}=\mathrm{e}^{P / \lambda_{*}}, \tau_{+}=\mathrm{e}^{P / \lambda_{+}}$.

Acknowledgements. This work was supported by the Belgian Science Policy Office through Prodex/CLUSTER (contract 13127/98/NL/VJ(IC)) and the Belgian Solar-Terrestrial Centre of Excellence.

Topical Editor R. Nakamura thanks two anonymous referees for their help in evaluating this paper. 


\section{References}

Chappell, C. R., Moore, T. E., and Waite Jr., J. H.: The ionosphere as a fully adequate source of plasma for the Earth's magnetosphere, J. Geophys. Res., 92, 5896-5910, doi:10.1029/ JA092iA06p05896, 1987.

De Keyser, J.: Formation and evolution of subauroral ion drifts in the course of a substorm, J. Geophys. Res., 104, 12339-12350, 1999.

De Keyser, J. and Echim, M.: Auroral and sub-auroral phenomena: an electrostatic picture, Ann. Geophys., 28, 633-650, doi:10.5194/angeo-28-633-2010, 2010.

De Keyser, J., Roth, M., Tsurutani, B., Ho, C., and Phillips, J.: Solar wind velocity jumps across tangential discontinuities: Ulysses observations and kinetic interpretation, A\&A, 321, 945-959, 1997.

Echim, M. M., Roth, M., and De Keyser, J.: Sheared magnetospheric plasma flows and discrete auroral arcs: a quasi-static coupling model, Ann. Geophys., 25, 317-330, doi:10.5194/angeo25-317-2007, 2007.

Echim, M. M., Roth, M., and De Keyser, J.: Ionospheric feedback effects on the quasi-stationary coupling between LLBL and postnoon/evening discrete auroral arcs, Ann. Geophys., 26, 913-928, doi:10.5194/angeo-26-913-2008, 2008.

Echim, M. M., Maggiolo, R., Roth, M., and De Keyser, J.: A magnetospheric generator driving ion and electron acceleration and electric currents in a discrete auroral arc observed by Cluster and DMSP, Geophys. Res. Lett., 36, L12111, doi:10.1029/2009GL038343, 2009.

Eliasson, L., André, M., Eriksson, A., Norqvist, P., Norberg, O., Lundin, R., Hollback, B., Koskinen, H., Borg, H., and Boehm, M.: Freja observations of heating and precipitation of positive ions, Geophys. Res. Lett., 21, 1911-1914, 1994.

Engwall, E., Eriksson, A. I., André, M., Dandouras, I., Paschmann, G., Quinn, J., and Torkar, K.: Low-energy (order $10 \mathrm{eV}$ ) ion flow in the magnetotail lobes inferred from spacecraft wake observations, Geophys. Res. Lett., 33, L06110, doi:10.1029/ 2005GL025179, 2006.

Figueiredo, S., Marklund, G. T., Karlsson, T., Johansson, T., Ebihara, Y., Ejiri, M., Ivchenko, N., Lindqvist, P.-A., Nilsson, H., and Fazakerley, A.: Temporal and spatial evolution of discrete auroral arcs as seen by Cluster, Ann. Geophys., 23, 2531-2557, doi:10.5194/angeo-23-2531-2005, 2005.

Gosling, J. T., Baker, D. N., Bame, S. J., Feldman, W. C., and Smith, E. J.: North-south and dawn-dusk plasma asymmetries in the distant tail lobes - ISEE 3, J. Geophys. Res., 90, 6354-6360, 1985.

Haaland, S., Paschmann, G., Frster, M., Quinn, J., Torbert, R., Vaith, H., Puhl-Quinn, P., and Kletzing, C.: Plasma convection in the magnetotail lobes: statistical results from Cluster EDI measurements, Ann. Geophys., 26, 2371-2382, doi:10.5194/angeo26-2371-2008, 2008.

Johansson, T., Karlsson, T., Marklund, G., Figueiredo, S., Lindqvist, P.-A., and Buchert, S.: A statistical study of intense electric fields at 4-7 RE geocentric distance using Cluster, Ann. Geophys., 23, 2579-2588, doi:10.5194/angeo-23-25792005, 2005.

Johansson, T., Marklund, G., Karlsson, T., Liléo, S., Lindqvist, P.-A., Marchaudon, A., Nilsson, H., and Fazakerley, A.: On the profile of intense high-altitude auroral electric fields at magnetospheric boundaries, Ann. Geophys., 24, 1713-1723, doi:10.5194/angeo-24-1713-2006, 2006.

Johansson, T., Marklund, G., Karlsson, T., Liléo, S., Lindqvist, P.A., Nilsson, H., and Buchert, S.: Scale sizes of intense auroral electric fields observed by Cluster, Ann. Geophys., 25, 24132425, doi:10.5194/angeo-25-2413-2007, 2007.

Kullen, A., Buchert, S., Karlsson, T., Johansson, T., Lileo, S., Eriksson, A., Nilsson, H., Marchaudon, A., and Fazakerley, A. N.: Plasma transport along discrete auroral arcs and its contribution to the ionospheric plasma convection, Ann. Geophys., 26, 32793293, doi:10.5194/angeo-26-3279-2008, 2008.

Lyons, L. R.: Generation of large-scale regions of auroral currents, electric potentials and precipitation by the divergence of the convection electric field, J. Geophys. Res., 85, 17-24, 1980.

Lyons, L. R.: Discrete aurora as the direct result of an inferred high altitude generating potential distribution, J. Geophys. Res., 86, $1-8,1981$.

Maggiolo, R., Sauvaud, J. A., Fontaine, D., Teste, A., Grigorenko, E., Balogh, A., Fazakerley, A., Paschmann, G., Delcourt, D., and Rème, H.: A multi-satellite study of accelerated ionospheric ion beams above the polar cap, Ann. Geophys., 24, 1665-1684, doi:10.5194/angeo-24-1665-2006, 2006.

Marghitu, O., Hamrin, M., Klecker, B., Vaivads, A., McFadden, J., Buchert, S., Kistler, L. M., Dandouras, I., André, M., and Rème, H.: Experimental investigation of auroral generator regions with conjugate Cluster and FAST data, Ann. Geophys., 24, 619-635, doi:10.5194/angeo-24-619-2006, 2006.

Marklund, G., Ivchenko, N., Karlsson, T., Fazakerley, A., Dunlop, M., Lindqvist, P.-A., Buchert, S., Owen, C., Taylor, M., Vaivads, A., Carter, P., André, M., and Balogh, A.: Temporal evolution of the electric field accelerating electrons away from the auroral ionosphere, Nature, 414, 724-727, 2001.

Moore, T. E., Lundin, R., Alcayde, D., André, M., Ganguli, S. B., Temerin, M., and Yau, A.: Source processes in the high-altitude ionosphere, Space Sci. Rev., 88, 7-84, 1999.

Rönnmark, K. and Hamrin, M.: Auroral electron acceleration by Alfvén waves and electrostatic field, J. Geophys. Res., 105, 25333-25344, 2000.

Roth, M., Evans, D. S., and Lemaire, J.: Theoretical structure of a magnetospheric plasma boundary: Application to the formation of discrete auroral arcs, J. Geophys. Res., 98, 11411-11423, 1993.

Roth, M., De Keyser, J., and Kuznetsova, M. M.: Vlasov theory of the equilibrium structure of tangential discontinuities in space plasmas, Space Sci. Rev., 76, 251-317, 1996.

Sestero, A.: Structure of plasma sheaths, Phys. Fluids, 7, 44-51, 1964.

Sestero, A.: Vlasov equation study of plasma motion across magnetic fields, Phys. Fluids, 9, 2006-2013, 1966.

Sigsbee, K., Cattell, C. A., Lysak, R. L., Carlson, C. W., Ergun, R. E., McFadden, J. P., Mozer, F., Elphic, R. C., Strangeway, R. J., Tsuruda, K., Yamamoto, T., Kokubun, S., Fairfield, D., Pfaff, R., Parks, G., and Brittnacher, M.: FAST-Geotail correlative studies of magnetosphere ionosphere coupling in the nightside magnetosphere, Geophys. Res. Lett., 25, 2077-2080, 1998.

Swift, D. W.: New perspectives on auroral morphology, Space Science Rev., 95, 489-500, 2001.

Temerin, M. and Carlson, C.: Current-voltage relationship in the downward auroral current region, Geophys. Res. Lett., 25, 23652368, 1998. 
Vaivads, A., André, M., Buchert, S., Eriksson, A. I., Olsson, A., Wahlund, J.-E., Janhunen, P., Marklund, G., Kistler, L. M., Mouikis, C., Winningham, D., Fazakerley, A., and Newell, P.: What high altitude observations tell us about the auroral acceleration: A Cluster/DMSP conjunction, Geophys. Res. Lett., 30, 1106, doi:10.1029/2002GL016006, 2003.
Vedin, J. and Rönnmark, K.: Electrostatic potentials in the downward auroral current region, J. Geophys. Res., 110, A08207, doi:10.1029/2005JA011083, 2005.

Walterscheid, R. L. and Lyons, L. R.: The neutral circulation in the vicinity of a stable auroral arc, J. Geophys. Res., 97, 1948919499, 1992. 